TRANSACTIONS OF THE

AMERICAN MATHEMATICAL SOCIETY

Volume 363, Number 12, December 2011, Pages 6713-6741

S 0002-9947(2011)05449-8

Article electronically published on June 3, 2011

\title{
SYMMETRIC TOPOLOGICAL COMPLEXITY AS THE FIRST OBSTRUCTION IN GOODWILLIE'S EUCLIDEAN EMBEDDING TOWER FOR REAL PROJECTIVE SPACES
}

\author{
JESÚS GONZÁLEZ
}

\begin{abstract}
As a first goal, it is explained why the Goodwillie-Weiss calculus of embeddings offers new information about the Euclidean embedding dimension of $\mathrm{P}^{m}$ only for $m \leq 15$. Concrete scenarios are described in these low-dimensional cases, pinpointing where to look for potential - but criticalhigh-order obstructions in the corresponding Taylor towers. For $m \geq 16$, the relation $\mathrm{TC}^{S}\left(\mathrm{P}^{m}\right) \geq n$ is translated into the triviality of a certain cohomotopy Euler class which, in turn, becomes the only Taylor obstruction to producing an embedding $\mathrm{P}^{m} \subset \mathbb{R}^{n}$. A speculative bordism-type form of this primary obstruction is proposed as an analogue of Davis' $B P$-approach to the immersion problem of $\mathrm{P}^{m}$. A form of the Euler class viewpoint is applied to show that $\mathrm{TC}^{S}\left(\mathrm{P}^{3}\right)=5$, as well as to suggest a few higher-dimensional projective spaces for which the method could produce new information. As a second goal, the paper extends Farber's work on the motion planning problem in order to develop the notion of a symmetric motion planner for a mechanical system $\mathcal{S}$. Following Farber's lead, this concept is connected to $\mathrm{TC}^{S}(C(\mathcal{S}))$, the symmetric topological complexity of the state space of $\mathcal{S}$. The paper ends by sketching the construction of a concrete 5-local-rules symmetric motion planner for $\mathrm{P}^{3}$.
\end{abstract}

\section{MAin RESUlts}

Recall the Schwarz genus $\mathfrak{g e n u s}(p)$ of a fibration $p: E \rightarrow B([26)$; it is one les: 1 than the smallest number of open sets $U$ covering $B$ in such a way that $p$ admits a (continuous) section over each $U$.

Definition $1.1([8)$. The topological complexity of a space $X, \operatorname{TC}(X)$, is defined as the genus of the end-points evaluation map ev: $P(X) \rightarrow X \times X$, where $P(X)$ is the free path space $X^{[0,1]}$ with the compact-open topology.

Let $F(X, 2) \subset X \times X$ denote the configuration space of ordered pairs of distinct points in $X$, and ev $: P_{1}(X) \rightarrow F(X, 2)$ be the restriction of the fibration ev. Thus $P_{1}(X)$ is the subspace of $P(X)$ obtained by removing the free loops on $X$. The group $\mathbb{Z} / 2$ acts freely on both $P_{1}(X)$ and $F(X, 2)$, by running a path backwards in

Received by the editors February 10, 2010 and, in revised form, August 11, 2010.

2010 Mathematics Subject Classification. Primary 57R40, 55M30, 55R80, 70E60.

Key words and phrases. Topological complexity, calculus of embeddings, configuration space.

The author was partially supported by CONACYT Research Grant 102783.

${ }^{1}$ Normalization is chosen so that a trivial fibration has $\mathfrak{g e n u s}=0$.

(C)2011 American Mathematical Society Reverts to public domain 28 years from publication 
the former, and by switching coordinates in the latter. Furthermore, ev $\mathrm{v}_{1}$ is a $\mathbb{Z} / 2$ equivariant map. Let $P_{2}(X)$ and $B(X, 2)$ denote the corresponding orbit spaces, and let $\mathrm{ev}_{2}: P_{2}(X) \rightarrow B(X, 2)$ denote the fibration induced by $\mathrm{ev}_{1}$.

Definition $1.2([10])$. The symmetric topological complexity of $X, \operatorname{TC}^{S}(X)$, is defined by $\operatorname{TC}^{S}(X)=\mathfrak{g e n u s}\left(\mathrm{ev}_{2}\right)+1$.

These constructions arise in connection with the motion planning problem in robotics. The reader interested in the motivation and further calculations should consult [8, 10] and the references therein.

The concept of a symmetric motion planner (as a collection of suitably chosen continuous $\mathbb{Z} / 2$-equivariant local sections - the local rules - for $\mathrm{ev}_{1}$ ) for a space, e.g. the state space of an autonomous robot, is formalized in Section 10 in order to prove:

Theorem 1.3. If $X$ is a smooth manifold, then $\mathrm{TC}^{S}(X)$ is the smallest possible number of local rules of symmetric motion planners for $X$.

The following result is derived in Section 9 by applying the ideas in Section 3

Theorem 1.4. $\mathrm{TC}^{S}(\mathrm{SO}(3))=5$. Consequently, 5 is the smallest possible number of local rules in any symmetric motion planner of an autonomous robot whose space of states is described by all three-dimensional rotations.

Section 10 describes an explicit 5-local-rules motion planner for an autonomous robot whose space of states is $\mathrm{SO}(3)$. The main ingredient in the proof of Theorem 1.4 comes from the following calculation, the main goal in Section 9

Theorem 1.5. The integral cohomology of $B(\mathrm{SO}(3), 2)$ is

$$
H^{*}(B(\operatorname{SO}(3), 2))= \begin{cases}\mathbb{Z}, & *=0 ; \\ 0, & *=1 \quad \text { or } * \geq 6 ; \\ \mathbb{Z} / 2 \oplus \mathbb{Z} / 2, & *=2 ; \\ \mathbb{Z} \oplus \mathbb{Z} / 2, & *=3 ; \\ \mathbb{Z} / 4, & *=4 ; \\ \mathbb{Z} / 2, & *=5 .\end{cases}
$$

Furthermore, the classifying map of the double cover $F(\mathrm{SO}(3), 2) \rightarrow B(\mathrm{SO}(3), 2)$ pulls back the square of the generator in $H^{2}\left(\mathrm{P}^{\infty}\right)$ to the element of order 2 in $H^{4}(B(\mathrm{SO}(3), 2))$.

Remark 1.6. Note that $H^{*}(B(\mathrm{SO}(3), 2))$ has both 2-torsion and 4-torsion, but no 8 -torsion. Fred Cohen has indicated to the author (even before the latter completed the proof of Theorem 1.5) that this seems to be the situation for all $B\left(\mathrm{P}^{m}, 2\right)$ with $m>2$. Here and in what follows, $\mathrm{P}^{m}$ stands for the $m$-dimensional real projective space. Cohen's conjecture has been recently verified for $m \not \equiv 3 \bmod 4$ in an ongoing project of the author with Peter Landweber (16]).

Some of the properties in the multiplicative structure of the cohomology ring of $B(\mathrm{SO}(3), 2)$ are discussed in Remark 9.8 .

Theorem 1.4 should be compared to the known equality $\mathrm{TC}(\mathrm{SO}(3))=3$. Actually, $\operatorname{TC}(G)=\operatorname{cat}(G)$ for any connected topological group $G$ (9]). On the other hand, it is known that $\operatorname{TC}^{S}(\mathrm{SO}(2))=2$ (see Table 1) while $\mathrm{TC}(\mathrm{SO}(2))=1$. An 
interesting open question arises, then, in regard to the deviation of $\mathrm{TC}^{S}$ from $\mathrm{TC}$ for a general connected topological (or even Lie) group. It should be noted that the inequality $\mathrm{TC}^{S} \geq \mathrm{TC}$ is known from [10].

Remark 8.11 in Section 8 provides some indirect evidence toward the possibility that the method of proof of Theorem 1.4 could actually be used in order to compute $\mathrm{TC}^{S}\left(\mathrm{P}^{m}\right)$ for (at least) $m=5,6$. The calculations in Section 9 (see in particular Remark 9.2) seem to suggest that the potential phenomenon mentioned in Remark 1.6 would play a critical role in the expected derivation of new information about $\mathrm{TC}^{S}\left(\mathrm{P}^{m}\right)$. In fact, the author hopes that this paper motivates a renewed interest in understanding the homotopy properties of the unordered configuration spaces $B\left(\mathrm{P}^{m}, 2\right)$, which are key objects in the Euclidean embedding problem for real projective spaces.

\section{TC, TC ${ }^{S}$, AND CAlCulus OF FUnCtors of EMBEDdings}

Despite their robotics origin, the topological complexity ideas are closely related, in the case of $\mathrm{P}^{m}$, to the calculus of embeddings developed in [19, 29] by Goodwillie and Weiss. This is one of the motivations for the present paper. The explicit connection is explained right after taking a quick glance at Goodwillie-Weiss' framework.

For a smooth $m$-dimensional manifold $M$, let $\mathcal{E}_{n}^{M}=\operatorname{Emb}\left(-, \mathbb{R}^{n}\right)$ denote the functor of smooth embeddings of open sets of $M$ into $\mathbb{R}^{n}$. The Taylor expansion of $\mathcal{E}_{n}^{M}$ is a tower of functors

$$
\cdots \rightarrow \mathcal{T}_{k} \mathcal{E}_{n}^{M} \stackrel{r_{k}}{\rightarrow} \ldots \stackrel{r_{3}}{\rightarrow} \mathcal{T}_{2} \mathcal{E}_{n}^{M} \stackrel{r_{2}}{\rightarrow} \mathcal{T}_{1} \mathcal{E}_{n}^{M}
$$

equipped with compatible mappings $\eta_{k}: \mathcal{E}_{n}^{M} \rightarrow \mathcal{T}_{k} \mathcal{E}_{n}^{M}$ which are to be thought of as giving better and better (as $k$ increases) approximations of $\mathcal{E}_{n}^{M}$. For instance, the standard model for the linear approximation $\mathcal{T}_{1} \mathcal{E}_{n}^{M}$ is the sheaf of smooth immersions into $\mathbb{R}^{n}$. In this case $\eta_{1}$ is the obvious inclusion, an equivalence through dimensions $n-2 m+1$ (Whitney's stable range). Likewise, for a manifold $M$ with

$$
2 n \geq 3(m+1)
$$

(recall $\operatorname{dim}(M)=m$ ), Haefliger's work shows that a model for the quadratic approximation $\mathcal{T}_{2} \mathcal{E}_{n}^{M}(M)$ is given by the space $\operatorname{Map}^{\mathbb{Z} / 2}\left(F(M, 2), S^{n-1}\right)$ of (continuous) $\mathbb{Z} / 2$-equivariant maps $F(M, 2) \rightarrow S^{n-1}$ (with antipodal $\mathbb{Z} / 2$-action on the sphere $\left.S^{n-1}\right)$. In these terms, $\eta_{2}(f)$ sends a pair $(x, y) \in F(M, 2)$ into the normalized difference

$$
\frac{f(x)-f(y)}{\|f(x)-f(y)\|} \in S^{n-1}
$$

determining an equivalence $\eta_{2}$ through dimensions $2 n-3 m-3$. More generally, in a certain range, the tower (10) is an analytic approximation for $\mathcal{E}_{n}^{M}$ since, according to [17, 19, for $n>m+2$,

$$
\eta_{k} \text { is an equivalence through dimensions } k n-(k+1) m-2 k+1 \text {. }
$$

Throughout the rest of this section, $M$ is replaced by the $m$-dimensional real projective space $\mathrm{P}^{m}$. The first point to explain is how the work in [11, 15] (which are the basic references for the following assertions) allows us to think of TC and 
$\mathrm{TC}^{S}$ as giving detecting indicators for the linear and quadratic terms in the Taylor tower for $\mathcal{E}_{n}^{\mathrm{P}^{m}}$. To begin with, according to [1], if $\mathcal{T}_{1} \mathcal{E}_{n}^{\mathrm{P}^{m}}\left(\mathrm{P}^{m}\right)$ is nonempty, then

$$
\mathrm{TC}\left(\mathrm{P}^{m}\right) \leq n
$$

Likewise, if $\mathcal{T}_{2} \mathcal{E}_{n}^{\mathrm{P}^{m}}\left(\mathrm{P}^{m}\right)$ is nonempty, then [15] gives

$$
\operatorname{TC}^{S}\left(\mathrm{P}^{m}\right) \leq n .
$$

Furthermore, not only do both implications hold without range restrictions but, except for a very limited number of cases, they are sharp. For instance, the implication involving (5) is reversible for a nonparallelizable $\mathrm{P}^{m}$, and in the three exceptional cases of parallelizability $(m=1,3,7)$ one has

$$
\mathcal{T}_{1} \mathcal{E}_{n}^{\mathrm{P}^{m}}\left(\mathrm{P}^{m}\right) \neq \varnothing \quad \Leftrightarrow \quad \mathrm{TC}\left(\mathrm{P}^{m}\right) \leq n-1 .
$$

The discussion of the situation for the implication involving (6) - the main topic in [15] - is perhaps more interesting. Namely, such an implication is reversible, for instance, when $\operatorname{TC}^{S}\left(\mathrm{P}^{m}\right)$ is within Haefliger's metastable range (2), that is, when

$$
2 \mathrm{TC}^{S}\left(\mathrm{P}^{m}\right) \geq 3(m+1) .
$$

In fact, Haefliger's work give:2

$$
\operatorname{Emb}\left(\mathrm{P}^{m}, \mathbb{R}^{n}\right) \neq \varnothing \Leftrightarrow \mathcal{T}_{2} \mathcal{E}_{n}^{\mathrm{P}^{m}}\left(\mathrm{P}^{m}\right) \neq \varnothing \Leftrightarrow \mathrm{TC}^{S}\left(\mathrm{P}^{m}\right) \leq n
$$

whenever (77) holds, so that $\mathrm{TC}^{S}\left(\mathrm{P}^{m}\right)$ agrees with $\operatorname{Emb}\left(\mathrm{P}^{m}\right)$, the embedding dimension of $\mathrm{P}^{m}$. In other words, within the range determined by (7), if a given immersion $f: \mathrm{P}^{m} \rightarrow \mathbb{R}^{n}$ can be regularly deformed into one coming from an element in $\mathcal{T}_{2} \mathcal{E}_{n}^{\mathrm{P}^{m}}\left(\mathrm{P}^{m}\right)$, then the immersion can be regularly deformed into an embedding. An alternative phrasing (which ignores indeterminacy issues) of this fact goes as follows: When (17) holds, there is only one obstruction to lifting elements in $\mathcal{T}_{1} \mathcal{E}_{n}^{\mathrm{P}^{m}}\left(\mathrm{P}^{m}\right)$ through the several maps $r_{k}$ in (1); that obstruction holds at $k=2$, has to do with avoiding double points, and is numerically detected by the condition $\mathrm{TC}^{S}\left(\mathrm{P}^{m}\right) \leq n$.

It is known from [15, that Haefliger's metastable range condition (7) holds for $m \geq 16$, as well as for $m=4,8,9,10,13$ (for the case $m=10$, see Table 1 and the comments in Section 7). But the equality $\mathrm{TC}^{S}\left(\mathrm{P}^{m}\right)=\operatorname{Emb}\left(\mathrm{P}^{m}\right)$ is also known for $m \leq 2$ and, from Theorem 1.4, for $m=3$. However, it is currently unknown if in any of the remaining cases,

$$
m \in\{5,6,7,11,12,14,15\},
$$

(a) the implication involving (6) is reversible,

(b) there are higher obstructions to lifting through the corresponding tower (1), or if, on the contrary,

(c) $\mathrm{TC}^{S}\left(\mathrm{P}^{m}\right)$ agrees with the embedding dimension of $\mathrm{P}^{m}$.

The major goal in this paper is to suggest ways to shed light on these lowdimensional cases, mainly through Projects 1 and 2 below, although Project 3 has interesting potential implications for higher-dimensional projective spaces (see item II in Section [5).

\footnotetext{
${ }^{2}$ Brian Munson's help, starting at the 2009 CBMS conference in Cleveland, has been fundamental for realizing the correct form of this fact.
} 
The section closes by recording the main theorem in [15], namely, the result of peeling Haefliger's numerical restriction (7) off the relation between $\mathcal{T}_{2} \mathcal{E}_{n}^{\mathrm{P}^{m}}\left(\mathrm{P}^{m}\right)$ and $\mathrm{TC}^{S}\left(\mathrm{P}^{m}\right)$. Explicit computations for $m=3$ are given at the end of Section 10 .

Theorem 2.1 ([15, Theorem 1.4]). $\operatorname{Map}^{\mathbb{Z} / 2}\left(F\left(\mathrm{P}^{m}, 2\right), S^{n-1}\right) \neq \varnothing$ if and only if $\mathrm{TC}^{S}\left(\mathrm{P}^{m}\right) \leq n$.

Remark 2.2. Haefliger's map (3) $, \eta_{2}: \operatorname{Emb}\left(M, \mathbb{R}^{n}\right) \rightarrow \operatorname{Map}^{\mathbb{Z} / 2}\left(F(M, 2), S^{n-1}\right)$, implies that the inequality $\mathrm{TC}^{S}\left(\mathrm{P}^{m}\right) \leq n$ is a consequence of the existence of an embedding $\mathrm{P}^{m} \subset \mathbb{R}^{n}$.

The rest of the paper is organized as follows. Sections 36 describe four projects aiming to get a hold on $\mathrm{TC}^{S}\left(\mathrm{P}^{m}\right)$ and $\operatorname{Emb}\left(\mathrm{P}^{m}\right)$. Potential examples on the use of these projects are given in Section 7 The reader interested in the proof of Theorems 1.4 and 1.5 should proceed directly to Section 9, going back as needed (explicit cross-references are given) to Section 3 for the general strategy, and to Section 8 for a preliminary $\mathbb{F}_{2}$-cohomology calculation due to Haefliger. The final section, Section 10, is devoted to (a) developing the concept of a symmetric motion planner for a mechanical system, (b) studying its relation with the symmetric topological complexity of the space of states of the system (proof of Theorem 1.3), and (c) sketching the construction of a concrete symmetric motion planner, with the least possible number of local rules, when the state space of the system is $\operatorname{SO}(3)$.

\section{THE FIRST PROJECT}

Theorem 2.1 can be restated by saying that $\mathrm{TC}^{S}\left(\mathrm{P}^{m}\right)$ is the smallest positive integer $n$ such that the classifying map for the line bundle $\zeta_{m}$ associated to the canonical $\mathbb{Z} / 2$-principal projection $F\left(\mathrm{P}^{m}, 2\right) \rightarrow B\left(\mathrm{P}^{m}, 2\right)$ admits a homotopy compression

$$
\zeta: B\left(\mathrm{P}^{m}, 2\right) \rightarrow \mathrm{P}^{n-1}
$$

A Hopf-type approach to this problem is then given by analyzing, with mod 2 singular cohomology, the algebraic possibilities for a potential map (91). Namely, the vanishing of the $n$-th power of $w_{1}\left(\zeta_{m}\right)$ is a necessary condition for the existence of a compressed map $\zeta$ as in (9), and thus for an embedding $\mathrm{P}^{m} \subset \mathbb{R}^{n}$. The method was known in the 1960s ([21]) and, during the subsequent decade, it was systematically used to evaluate, within Haefliger's metastable range, groups of embeddings of low efficiency ([3, 12, 31, 32]) for a given manifold $M$. The main input for those computations (and their answer) is given by the integral cohomology groups of $B(M, 2)$ with both simple and local coefficients.

The interest in this section focuses on $M=\mathrm{P}^{m}$, keeping more of the original motivation in [21], but without having to restrict attention to Haefliger's metastable range (77). For instance, the explicit cases worked out at the end of Section 8 allow us to recover, for $m \leq 8$, the lower bounds for $\mathrm{TC}^{S}\left(\mathrm{P}^{m}\right)$ obtained in [15] using ideas in [10. But integral coefficient cohomology rings are now central, and even more interesting is the possibility that, by replacing singular cohomology by suitable generalized cohomology theories, one could get important improvements on the lower bounds for $\mathrm{TC}^{S}\left(\mathrm{P}^{m}\right)$, which, in view of Remark 2.2 might even lead to new nonembedding results for $\mathrm{P}^{m}$. A number of potential situations of this sort are described in Section 7. The idea is in the same spirit as the one exploited in [1, 5] 
(for immersions rather than embeddings) using forms of complex cobordism instead of singular cohomology; compare to item II in Section 5 .

In this direction, the first proposed project is:

\section{Project 1.}

(1) Describe (enough of) the integral cohomology ring $H^{*}\left(B\left(\mathrm{P}^{m}, 2\right)\right)$.

(2) (Profit:) Apply the resulting information within the Hopf-type approach described right after (9) to get (new, perhaps optimal in certain low-dimensional cases) lower bounds for $\mathrm{TC}^{S}\left(\mathrm{P}^{m}\right)$ and potentially for $\operatorname{Emb}\left(\mathrm{P}^{m}\right)$.

The case of $\bmod p$ coefficients in part 1 of Project 1 was treated by Haefliger [20] $(p=2)$ and Félix-Tanré [13] (odd prime $p)$. Haefliger's method is reviewed in Section 8. The information is used in Section 9, following the ideas in Project 1, to compute $\mathrm{TC}^{S}\left(\mathrm{P}^{3}\right)$.

As far as the author knows, the integral cohomology ring - or, for that matter, its additive structure - required in part 1 of Project 1 is currently unknown (3] gives a theoretical description of the Bockstein spectral sequence for $B\left(\mathrm{P}^{m}, 2\right)$ when $m$ is odd, but the explicit calculations get combinatorially out of hand as $m$ increases). Information about this ring would in addition pave the way for the corresponding calculation in terms of some (advantageous for the Hopf-type approach) generalized cohomology theory, e.g. complex cobordism. Furthermore, information on the integral cohomology ring $H^{*}\left(B\left(\mathrm{P}^{m}, 2\right)\right)$ is not only usable as described in Project 1 . but it would lay the groundwork for Project 2, which is presented next.

\section{THE SECOND PROJECT}

Since $\mathrm{P}^{n-1}$ classifies line bundles whose $n$-th Whitney multiple admits a nowhere zero section (14), the considerations in (9) imply that Theorem 2.1 can be recast in the following terms: $\mathrm{TC}^{S}\left(\mathrm{P}^{m}\right)$ is the smallest positive integer $n$ such that $n \zeta_{m}$ admits a nowhere zero section. In particular, the Hopf-type approach in the previous project can be interpreted in terms of the observation that (generalized) Euler classes give initial obstructions to sectioning $n \zeta_{m}$ (indeed, this is the starting point in the classical obstruction theory approach of [21]). For a given cohomology theory $E$, much of the problem in the Euler class setting comes from the fact that, due to a possible lack of $E$-orientability of $n \zeta_{m}$ (or $\zeta_{m}$, for that matter), the calculation of the $E$-Euler class $\chi_{E}\left(n \zeta_{m}\right)$ might become a very difficult task, but note that generalized Euler classes are defined even without orientability assumptions (4]). This difficulty is largely redeemed by the nice fact that the cohomotopy Euler class is the unique obstruction for the mono-sectioning problem of bundles of relatively large dimension. Concretely, as indicated in 4, a bundle $\alpha$ over a complex $X$ satisfying

$$
2 \operatorname{dim}(\alpha) \geq \operatorname{dim}(X)+3
$$

admits a nowhere zero section if and only if the cohomotopy Euler class $\chi_{S^{0}}(\alpha)$ vanishes. This fact is very effectively put to work by Stolz in [27] in order to give a complete description of the first Whitney multiple of $\lambda_{m}$ admitting a nowhere zero section, where $\lambda_{m}$ is the canonical line bundle over a given $\mathrm{P}^{2 m+1}$.

Since an embedding $\mathrm{P}^{m} \subset \mathbb{R}^{n}$ can exist only with $n>m$, and since

(11) $B\left(\mathrm{P}^{m}, 2\right)$ has the homotopy type of a closed $(2 m-1)$-dimensional manifold 
(see [21, Proposition 2.6]), condition (10) holds in all relevant instances of $\alpha=n \zeta_{m}$. This situation can now be used to complement Project 1 whose long term goal can be thought of as finding suitable cohomology theories $E$, where $\chi_{E}\left(n \zeta_{m}\right) \neq 0$ (thus obtaining lower bounds for $\mathrm{TC}^{S}\left(\mathrm{P}^{m}\right)$ and, consequently, for $\left.\operatorname{Emb}\left(\mathrm{P}^{m}\right)\right)$. Indeed, the next project is concerned with getting upper bounds for $\mathrm{TC}^{S}\left(\mathrm{P}^{m}\right)$ by means of the exploration of the cohomotopy Euler class of $n \zeta_{m}$ for suitable values of $n$.

\section{Project 2 .}

(1) Adapt Stolz's method in order to identify instances where the cohomotopy Euler class of $n \zeta_{m}$ vanishes.

(2) (Profit:) This would produce upper bounds for the symmetric topological complexity of projective spaces, as well as (potentially new) embeddings of those manifolds in Haefliger's metastable range.

Stolz's method for studying cohomotopy Euler classes makes essential use of the calculability of the corresponding integral (singular) cohomology Euler classes. It is in this sense that the integral cohomology calculations needed for Project 1 would also find applications in Project 2.

\section{THE THIRD PROJECT}

While the numerical behavior of $\mathrm{TC}^{S}\left(\mathrm{P}^{m}\right)$ is the central issue in the first two projects, the next two will concern, instead, the higher obstructions one could meet in the Taylor tower (1) for $M=\mathrm{P}^{m}$. Thus, the next project can be thought of as an extension of (the cobordism approach in) Project 1 .

Start by recalling Hirsch's interpretation of the immersion problem for a closed manifold $M$ in terms of the geometric dimension of its stable normal bundle $\nu_{M}$ : the minimal codimension of Euclidean immersions of $M$ agrees with the smallest positive integer $d$ for which the map $M \rightarrow B O$ classifying $\nu_{M}$ admits a homotopy factorization

$$
M \rightarrow B O(d) \hookrightarrow B O .
$$

Obstruction theory then decomposes this task into small goals in which one has to deal with the $k$-invariants in a suitably flavored Postnikov tower for (a fibration homotopy equivalent to) the inclusion $B O(d) \hookrightarrow B O$. Such obstructions are theoretically easy to describe (they lie in singular cohomology groups), but are very hard to handle in practice (not only do they require information about the homotopy fiber of $B O(d) \hookrightarrow B O$, but obstructions can appear in the tower at a high level involving difficult indeterminacy issues). For real projective spaces, the latter problem was avoided in [5] (with remarkable results) by considering just one obstruction, a key Euler class in Brown-Peterson theory. This primary obstruction captures a great deal of information and can be handled efficiently by means of algebraic methods. Although there is a corresponding version of such an obstruction for the embedding (as opposed to immersion) dimension of projective spaces ([2]), the comments below (particularly item II) should be thought of as suggesting the possibility of a primary obstruction for the embedding problem of projective spaces, similar in spirit to that in [5], but now giving a complete obstruction within Haefliger's metastable range.

The question of identifying secondary (and higher-order) cobordism obstructions in the Euclidean embedding problem of a given manifold arises very naturally from 
the Taylor expansion point of view (in the context of regularly deforming a given immersion into an embedding). For instance, just as Haefliger pointed out the primary obstruction to lifting an element through $r_{2}$ in (11), the (secondary) obstruction to lifting through $r_{3}$ lies in a certain twisted cobordism group (24]). Now, as explained in the comments prior to Theorem 2.1. for projective spaces such higher obstructions can only appear in the cases (outside Haefliger's metastable range) indicated in (8). These assertions have two interesting (potential) consequences:

I. High-order analysis of obstructions (secondary obstructions in particular) in the Taylor tower (11) has good possibilities to detect new phenomena in the embedding problem of low-dimensional (read: outside Haefliger's metastable range) projective spaces; see the concrete situations worked out in Section 7

II. (Again with $M=\mathrm{P}^{m}$ ) If it is possible to identify the Brown-Peterson Euler class in the Hopf-type approach in Project 1 with Haefliger's obstruction for lifting through $r_{2}$ in (10), then this would mean that, within Haefliger's metastable range (e.g. for $m \geq 16$ ), this Brown-Peterson (primary) obstruction would be the only one for the embedding problem of projective spaces. It is very appealing to compare this possibility with the "feeling" that the negative immersion results in [5] seem to be closer to optimal than the general positive immersion results currently known for projective spaces. But the advantage in the new proposed embedding setting is that the resulting obstruction would be complete, thus providing formal support for the embedding analogue of the feeling mentioned above.

The most difficult part in the previous setting would seem to be the identification (and manipulation) of the cobordism groups containing high-order obstructions (not to mention the actual calculation of the obstructions). Thus, the new project is:

\section{Project 3.}

(1) (In the direction of I above:) Give an explicit (= manageable) description (recall $M=\mathrm{P}^{m}$ ) of the cobordism group containing the obstruction for lifting, through $r_{3}$, a given element in $\mathcal{T}_{2} \mathcal{E}_{n}^{\mathrm{P}^{m}}\left(\mathrm{P}^{m}\right)$. Since this question is intended for values of $m$ outside Haefliger's metastable range, the required model for $\mathcal{T}_{2} \mathcal{E}_{n}^{\mathrm{P}^{m}}\left(\mathrm{P}^{m}\right)$ would not be the space $\operatorname{Map}^{\mathbb{Z} / 2}\left(F\left(\mathrm{P}^{m}, 2\right), S^{n-1}\right)$, but the usual space $\mathrm{Iso}^{\mathbb{Z} / 2}\left(\mathrm{P}^{m} \times \mathrm{P}^{m}, \mathbb{R}^{n} \times \mathbb{R}^{n}\right)$ of strict isovariant maps.

(2) Identify instances where this cobordism group vanishes.

(3) (Profit:) Except for a few very low-dimensional projective spaces (specified in Section 7), the resulting lifted map would be within the $3 / 4$ range ( $k=3$ in (44), so the corresponding embedding would be for free.

(4) (Profit:) On the other hand, if one could prove nontriviality of Munson's obstruction [24] for any strict isovariant map, then this would certainly imply a corresponding nonembedding result.

(5) (In the direction of II above:) Sort out the hoped-for identification of primary obstructions mentioned in II above. If this does not work, then find an algebraic characterization (some sort of cobordism group?) of Haefliger's double obstruction for lifting through $r_{2}$-(profit:) such an obstruction is then the only one, within Haefliger's metastable range, for the embedding problem of projective spaces. 
TABle 1. $\operatorname{Emb}\left(\mathrm{P}^{m}\right)$ vs. $\mathrm{TC}^{S}\left(\mathrm{P}^{m}\right)$ for low values of $m$

\begin{tabular}{|c|c|c|c|c|c|c|c|c|c|c|c|c|c|c|c|}
\hline$m$ & 1 & 2 & 3 & $4^{\star}$ & 5 & 6 & 7 & $8^{\star}$ & $9^{\star}$ & $10^{\star}$ & 11 & 12 & $13^{\star}$ & 14 & 15 \\
\hline $\operatorname{Emb}\left(\mathrm{P}^{m}\right)$ & 2 & 4 & 5 & 8 & 9 & $9 . .11$ & $9 . .12$ & 16 & 17 & 17 & $17 . .18$ & $18 . .21$ & $22 . .23$ & $22 . .23$ & $23 . .24$ \\
\hline $\mathrm{TC}^{S}\left(\mathrm{P}^{m}\right)$ & 2 & 4 & $\mathbf{5}$ & 8 & $8 . .9$ & $8 . .9$ & $8 . .10$ & 16 & 17 & $\mathbf{1 7}$ & $\mathbf{1 7 . . 1 8}$ & $18 . .21$ & $22 . .23$ & $22 . .23$ & $22 . .23$ \\
\hline
\end{tabular}

For part 2 of Project 3 to make better sense, one would need to be specific about how to identify good "instances", i.e., those with high chances for Munson's secondary obstruction to vanish. Indeed, one needs to know where to look for potential isovariant maps (i.e. those in part 1 of Project 3) with trivial Munson's secondary obstruction. In view of Theorem 2.1 and Remark 2.2 a first approximation comes from the knowledge of the value of $\mathrm{TC}^{S}\left(\mathrm{P}^{m}\right)$ - the motivation for the two previous projects. As a first step in this direction, Section 7 describes what the author knows about concrete values of $\operatorname{TC}^{S}\left(\mathrm{P}^{m}\right)$ for low values of $m$, as well as about the expectations for high-order obstructions in (11), and possible ways to manage them by comparing with known information on the embedding dimension of lowdimensional projective spaces (indeed, functoriality issues might be helpful toward part 4 of Project 3 .

\section{The FOURTh PROJECT}

The last project is of a more theoretical nature: it has to do with finding models suitably "approximating" the terms $\mathcal{T}_{k} \mathcal{E}_{n}^{M}$ in (11). The idea is still in a very crude stage and is stated likewise.

Project 4. For $k \geq 3$, find a space that models $\mathcal{T}_{k} \mathcal{E}_{n}^{M}(M)$ in a way that resembles how $\operatorname{Map}^{\mathbb{Z} / 2}\left(F(M, 2), S^{n-1}\right)$ models $\mathcal{T}_{2} \mathcal{E}_{n}^{M}(M)$.

Haefliger-type models for $\mathcal{T}_{k} \mathcal{E}_{n}^{M}$ have certainly been described in [18, but the author does not know how manageable those models are for concrete computations (e.g. with $M=\mathrm{P}^{m}$ ). The idea here is to find alternative models which can be handled more naturally from an algebraic topology point of view, even if this means concentrating on $M=\mathrm{P}^{m}$.

\section{Putting the projects to Work}

Recall from Remark 2.2 that $\operatorname{Emb}\left(\mathrm{P}^{m}\right)$, the embedding dimension of $\mathrm{P}^{m}$, is an upper bound for $\mathrm{TC}^{S}\left(\mathrm{P}^{m}\right)$, and that equality actually holds when $\mathrm{TC}^{S}\left(\mathrm{P}^{m}\right)$ satisfies Haefliger's metastable range condition (7), e.g. when $m \geq 16$. This section starts by describing what the author knows about $\mathrm{TC}^{S}\left(\mathrm{P}^{m}\right)$ and $\operatorname{Emb}\left(\mathrm{P}^{m}\right)$ for low values of $m$ (potentially outside Haefliger's metastable range). The data is summarized in Table 1, where cases satisfying (7) have been marked with a star. The information is taken from [15. Table 1 on page 480], except for the boldface number 5 for $m=3$, and the two boldface numbers 17 (which, after the required shift in notation, appear as 16 in [15]) for $m=10,11$. The improvement for $m=3$ is given by Theorem 1.4, while those for $m=10,11$ follow from Theorem 2.1. the known case $\operatorname{TC}^{S}\left(\mathrm{P}^{9}\right)=17$, and the $\mathbb{Z} / 2$-equivariant inclusions $F\left(\mathrm{P}^{9}, 2\right) \hookrightarrow$ $F\left(\mathrm{P}^{10}, 2\right) \hookrightarrow F\left(\mathrm{P}^{11}, 2\right)$. 
In searching for exceptional cases of $m$, "exceptional" in the sense that the expected equality $\operatorname{TC}^{S}\left(\mathrm{P}^{m}\right)=\operatorname{Emb}\left(\mathrm{P}^{m}\right)$ fails, the situations to consider are:

Case $m=3$. This was the first undecided situation before this paper. The question was $([15])$ :

$$
\text { Is } \mathrm{TC}^{S}\left(\mathrm{P}^{3}\right)=4 \text {, or is } \mathrm{TC}^{S}\left(\mathrm{P}^{3}\right)=5 \text { ? }
$$

As shown in Section 9 the method in Project 1 allows one to show that the second possibility in (12) is the correct answer. Remark 8.11 suggests that it might be possible to resolve the cases $m=5$ and $m=6$ discussed below along the same lines.

Case $m=5$. The focus is on answering the question:

$$
\text { Is } \mathrm{TC}^{S}\left(\mathrm{P}^{5}\right)=8 \text {, or is } \mathrm{TC}^{S}\left(\mathrm{P}^{5}\right)=9 \text { ? }
$$

But there is an interesting subtlety not present in (12). Start by observing that the $(n=8)$-Taylor tower (11) for $\mathrm{P}^{5}$ is analytic and has a nonempty 3 starting space $\operatorname{Imm}\left(\mathrm{P}^{5}, \mathbb{R}^{8}\right)$, but that there must be a nontrivial obstruction since the embedding dimension of $\mathrm{P}^{5}$ is known to be 9 . Moreover, (4) implies that any such obstruction has to show up when trying to lift through $r_{2}, r_{3}$, or $r_{4}$. ( $\eta_{4}$ is an equivalence in the present situation.) But then a potential (exceptional) case $\operatorname{TC}^{S}\left(\mathrm{P}^{5}\right)=8$ does not rule out a possible scenario where the obstruction arises right at the very first lifting $r_{2}$; it would just mean that, in such a hypothetical situation, the space $\operatorname{Map}^{\mathbb{Z} / 2}\left(F\left(\mathrm{P}^{5}, 2\right), S^{7}\right)$ wouldn't be the right model for $\mathcal{T}_{2} \mathcal{E}_{8}^{\mathrm{P}^{5}}$. These possibilities have been explained in some detail since, from a different viewpoint, they could be used to actually produce new embeddings of higher-dimensional projective spaces, as discussed in the next cases.

Case $m=6$. Here the focus is on answering (13) with $\mathrm{P}^{6}$ replacing $\mathrm{P}^{5}$. Note that the exceptional situation with $\mathrm{TC}^{S}\left(\mathrm{P}^{6}\right)=8$ is not quite similar to that for $m=5$; there is a possible lack of analyticity now. However, there is a new interesting point coming from the fact that the explicit value of $\operatorname{Emb}\left(\mathrm{P}^{6}\right)$ is currently unknown. Namely, one could try to use the approach in Project 3 in order to construct new embeddings of $\mathrm{P}^{6}$. For instance, the simplest case would be to take $M=\mathrm{P}^{6}$ and $n=10$ in (11), trying to produce the (new ) embedding

$$
\mathrm{P}^{6} \subset \mathbb{R}^{10} \text {. }
$$

Although this situation is still not within Haefliger's metastable range, the known fact $\mathrm{TC}^{S}\left(\mathrm{P}^{6}\right) \leq 9$ seems to suggest that there shouldn't be any obstruction to lifting a given immersion $g: \mathrm{P}^{6} \rightarrow \mathbb{R}^{10}$ through the corresponding tower (11) to produce an element $\widetilde{g} \in \mathcal{T}_{2} \mathcal{E}_{10}^{\mathrm{P}^{6}}\left(\mathrm{P}^{6}\right)$. But it is even more interesting to note that since $\eta_{3}$ is an equivalence in our current range, there is just one obstruction for lifting $\widetilde{g}$ to the desired embedding (14): Munson's (secondary) obstruction. So (potential profit!), if there is no such secondary obstruction, then one would be left with the (previously unknown smooth) embedding (14).

\footnotetext{
${ }^{3}$ Since Imm $=\mathrm{TC} \leq \mathrm{TC}^{S}$, the nonempty starting space of immersions is also the case in all the remaining situations of the section.

${ }^{4}$ This could be interpreted as smoothing Rees' PL-embedding of $\mathrm{P}^{6}$ into $\mathbb{R}^{10}([25)$.
} 
Case $m=7$. This situation is entirely similar to the one in the previous case, with Project 3 being a potential tool for producing new embeddings of $\mathrm{P}^{7}$ (a possible embedding into $\mathbb{R}^{11}$, smoothing Rees', is now the new simplest case to try). But in this case there is one further intriguing feature, namely, the possibility of using the approach in Project 1 to prove $\mathrm{TC}^{S}\left(\mathrm{P}^{7}\right)>9$; this would imply the new nonembedding result $\mathrm{P}^{7} \not \subset \mathbb{R}^{9}$ (such a possible result would be 'strong' in view of the parallelizability of $\mathrm{P}^{7}$ ).

Case $m \geq 11$. In all these cases $\eta_{3}$ is an equivalence in the relevant range, so the considerations about using Project 3 as discussed around (14) apply here too (i.e. Munson's secondary obstruction could play an important role in constructing new embeddings for these $\mathrm{P}^{m}$ ).

Note that if one could settle the relation $\mathrm{TC}^{S}\left(\mathrm{P}^{11}\right)>17$, following, say, the guidelines in Project 1 then not only the actual value of $\mathrm{TC}^{S}\left(\mathrm{P}^{11}\right)$ would be settled, but it would also follow that $\operatorname{Emb}\left(\mathrm{P}^{11}\right)=18$; again, this would be a new result. Similar considerations apply to $\mathrm{P}^{13}$ and $\mathrm{P}^{14}$, as well as to $\mathrm{P}^{12}$ if one could provethe rather unlikely- $-\mathrm{TC}^{S}\left(\mathrm{P}^{12}\right)>20$. But it is interesting to note that a new embedding result, this time for $\mathrm{P}^{12}$, would also follow if one could actually prove $\mathrm{TC}^{S}\left(\mathrm{P}^{12}\right) \leq 20$; indeed, such an inequality would produce the new embedding $\mathrm{P}^{12} \subset \mathbb{R}^{20}$ in view of (2) and (7).

But perhaps one of the most fruitful cases to consider is that of $\mathrm{P}^{15}$, where functoriality properties could be exploited in an eventual analysis of obstructions to lift elements in Taylor towers. For instance, in the exceptional case that $\mathrm{TC}^{S}\left(\mathrm{P}^{15}\right)=$ 22 , any actual element $x \in \mathcal{T}_{2} \mathcal{E}_{22}^{\mathrm{P}^{15}}\left(\mathrm{P}^{15}\right)$ would necessarily have a nontrivial Munson's obstruction (because $\operatorname{Emb}\left(\mathrm{P}^{15}\right)>22$ ). Now, if such an obstruction were to depend only on the 14-th (resp. 13-th) skeleton of $\mathrm{P}^{15}$ (say by an explicit calculation), then the corresponding (restricted) element $\bar{x} \in \mathcal{T}_{2} \mathcal{E}_{22}^{\mathrm{P}^{14}}\left(\mathrm{P}^{14}\right)\left(\right.$ resp. $\left.\mathcal{T}_{2} \mathcal{E}_{22}^{\mathrm{P}^{13}}\left(\mathrm{P}^{13}\right)\right)$ would also have a nontrivial Munson secondary obstruction to lift to $\mathcal{T}_{3} \mathcal{E}_{22}^{\mathrm{P}^{14}}\left(\mathrm{P}^{14}\right)$ (resp. $\left.\mathcal{T}_{3} \mathcal{E}_{22}^{\mathrm{P}^{13}}\left(\mathrm{P}^{13}\right)\right)$. The point then is that, modulo the usual primary and secondary indeterminacy considerations, this could lead to the (again new, but now optimal) potential nonembedding result $\mathrm{P}^{14} \not \subset \mathbb{R}^{22}$ (respectively $\mathrm{P}^{13} \not \subset \mathbb{R}^{22}$ and $\left.\mathrm{P}^{14} \not \subset \mathbb{R}^{22}\right)$.

Finally, and although the following remark has been noted in a general form in the first paragraph of the case $m \geq 11$, let us observe that, in view of Rees' $\mathrm{PL}$ embedding $\mathrm{P}^{15} \subset \mathbb{R}^{23}$ ([25]), and since at any rate $\mathrm{TC}^{S}\left(\mathrm{P}^{15}\right) \leq 23$, Munson's secondary obstruction for an element in $\mathcal{T}_{2} \mathcal{E}_{23}^{\mathrm{P}^{15}}\left(\mathrm{P}^{15}\right)$ is the only obstruction to producing a potential embedding $\mathrm{P}^{15} \subset \mathbb{R}^{23}$; again, this would be a new result, optimal in fact, that would smooth Rees' PL embedding.

\section{Mod 2 Cohomology of $B\left(\mathrm{P}^{m}, 2\right)$}

This section starts with a description of Haefliger's method 20] for computing the cohomology ring $H^{*}\left(B(M, 2) ; \mathbb{F}_{2}\right)$ for a closed smooth $m$-dimensional manifold $M$ (see [10). This information is then analyzed for $M=\mathrm{P}^{m}$ in connection with some of the lower bounds for $\mathrm{TC}^{S}\left(\mathrm{P}^{m}\right)$ in Table 1 . Throughout this section, $\mathbb{F}_{2}$ stands for the field with 2 elements. The notation $\Sigma_{2}$ or $\mathbb{Z} / 2$ will also be used when referring to the group structure in $\mathbb{F}_{2}$. 
Start with the Borel construction $S^{\infty} \times_{\Sigma_{2}} M^{2}$, the total space in the standard fibration

$$
M^{2} \rightarrow S^{\infty} \times_{\Sigma_{2}} M^{2} \rightarrow \mathrm{P}^{\infty}
$$

where $\Sigma_{2}$ acts on $M^{2}$ by swapping factors. Steenrod showed (see [20, Subsection 2.4] for a sketch of a proof of this and the subsequent facts in this paragraph) that the mod 2 Serre spectral sequence for this fibration collapses, so that one gets a ring isomorphism

$$
H^{*}\left(S^{\infty} \times_{\Sigma_{2}} M^{2} ; \mathbb{F}_{2}\right) \cong H^{*}\left(\mathrm{P}^{\infty} ; H^{*}\left(M ; \mathbb{F}_{2}\right)^{\otimes 2}\right) .
$$

Here $\pi_{1}\left(\mathrm{P}^{\infty}\right)=\Sigma_{2}$ acts on $H^{*}\left(M ; \mathbb{F}_{2}\right)^{\otimes 2}$ by swapping factors. In particular the action is trivial on diagonal elements $x \otimes x$, whereas $x \otimes y$ and $y \otimes x$ generate a split free $\Sigma_{2}$-submodule if $x \neq y$ and $x \neq 0 \neq y$. Thus, fixing a basis $\left\{a_{r}\right\}$ of $H^{*}\left(M ; \mathbb{F}_{2}\right)$, (16) transforms into the ring isomorphism

$$
H^{*}\left(S^{\infty} \times_{\Sigma_{2}} M^{2} ; \mathbb{F}_{2}\right) \cong\left(\mathbb{F}_{2}[z] \otimes D\right) \oplus N,
$$

where $z$ is the image of the generator $z \in H^{1}\left(\mathrm{P}^{\infty} ; \mathbb{F}_{2}\right)$ under the projection map in (15), $D$ is additively generated by the diagonal elements $1 \otimes a_{r} \otimes a_{r}$ (also denoted by $a_{r} \otimes a_{r}$ ), and $N$ is additively generated by the $\Sigma_{2}$-invariant sums $a_{r} \otimes a_{s}+a_{s} \otimes a_{r}$ (with $r \neq s$ ). Note that (17) is an isomorphism of $\left(H^{*}\left(\mathrm{P}^{\infty} ; \mathbb{F}_{2}\right)=\mathbb{F}_{2}[z]\right)$-algebras, where $z$ acts trivially on $N$ but freely on $D$. In particular, the product of an element $z^{i} \otimes a_{r} \otimes a_{r} \in \mathbb{F}_{2}[z] \otimes D$ and an element $a_{s} \otimes a_{t}+a_{t} \otimes a_{s} \in N$ is trivial for $i>0$, whereas $\left(a_{r} \otimes a_{r}\right)\left(a_{s} \otimes a_{t}+a_{t} \otimes a_{s}\right)=a_{r} a_{s} \otimes a_{r} a_{t}+a_{r} a_{t} \otimes a_{r} a_{s}$ is easily seen to lie in $N$ (in particular, $N$ is a subring, but $D$ is not). Furthermore, the Steenrod algebra action on $H^{*}\left(M ; \mathbb{F}_{2}\right)$ is closely related to the diagonal map $\Delta: \mathrm{P}^{\infty} \times M=S^{\infty} \times_{\Sigma_{2}} M \rightarrow S^{\infty} \times_{\Sigma_{2}} M^{2}$, where $\Sigma_{2}$ acts trivially on $M$. Indeed, $\Delta^{*}$ is an $\mathbb{F}_{2}[z]$-algebra map vanishing on $N$ such that

$$
\Delta^{*}(x \otimes x)=1 \otimes x^{2}+z \otimes \mathrm{Sq}^{k-1} x+z^{2} \otimes \mathrm{Sq}^{k-2} x+\cdots+z^{k} \otimes x
$$

for $x \in H^{k}\left(M ; \mathbb{F}_{2}\right)$ (see for instance [22, page 500]).

To determine the multiplicative structure in $H^{*}\left(B(M, 2) ; \mathbb{F}_{2}\right)$, Haefliger considers the map induced in cohomology by the inclusion

$$
j: B(M, 2) \simeq S^{\infty} \times_{\Sigma_{2}} F(M, 2) \hookrightarrow S^{\infty} \times_{\Sigma_{2}} M^{2} .
$$

Of course $j^{*}$ is a ring morphism, but it turns out to be surjective. Therefore the multiplicative structure in $H^{*}\left(B(M, 2) ; \mathbb{F}_{2}\right)$ will be determined from that of (17) once $\operatorname{ker} j^{*}$ is described - in Theorem 8.1 below. First a little notation. Consider the push-forward map

$$
\Delta_{!}: H^{*-m}\left(M ; \mathbb{F}_{2}\right) \rightarrow H^{*}\left(M^{2} ; \mathbb{F}_{2}\right)
$$

induced by the diagonal embedding $\operatorname{diag}: M \hookrightarrow M \times M$. This is given by

$$
\Delta_{!}(x)=(1 \otimes x) \smile \delta=(x \otimes 1) \smile \delta,
$$

where $\delta \in H^{m}\left(M^{2} ; \mathbb{F}_{2}\right)$ is the diagonal cohomology class, the image of the fundamental class under the restriction map $H^{m}\left(M \times M, M \times M-\operatorname{diag} ; \mathbb{F}_{2}\right) \rightarrow$ $H^{m}\left(M \times M ; \mathbb{F}_{2}\right)$. The final piece of information Haefliger needs is given by the

\footnotetext{
${ }^{5}$ The author thanks Peter Landweber for indicating the fact that the correct definition of $D$ in [20] should be given in terms of a basis of $H^{*}\left(M ; \mathbb{F}_{2}\right)$, and by noticing that the resulting $N$ is independent of the chosen basis.
} 
(degree $m$ ) endomorphism $\varphi: H^{*}\left(\mathrm{P}^{\infty} \times M ; \mathbb{F}_{2}\right) \rightarrow H^{*}\left(\mathrm{P}^{\infty} \times M ; \mathbb{F}_{2}\right)$ given by multiplication by the class

$$
z^{m} \otimes w_{0}+z^{m-1} \otimes w_{1}+\cdots+1 \otimes w_{m},
$$

where $W=W_{M}=\sum_{i \geq 0}^{m} w_{i}$ is the total Stiefel-Whitney class of $M$.

Theorem 8.1 (Haefliger 20]). There exists a commutative diagram with exact rows:

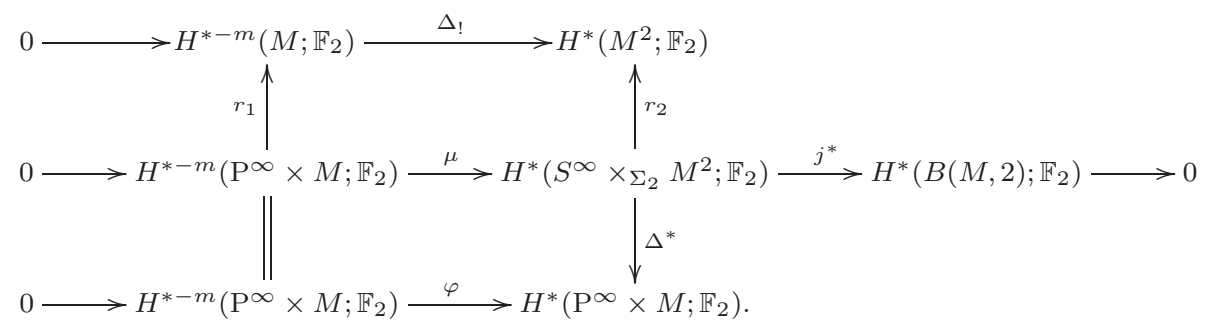

Here $r_{2}$ is induced by the fiber inclusion in (15); the situation for $r_{1}$ is similar (in terms of the identification $\left.\mathrm{P}^{\infty} \times M=S^{\infty} \times_{\Sigma_{2}} M\right)$.

Remark 8.2. As an easy consequence of the fact that $r_{2}$ is monic on $N=\operatorname{ker} \Delta^{*}$, one finds that every Steenrod square $\mathrm{Sq}^{i}: H^{*}\left(S^{\infty} \times_{\Sigma_{2}} M^{2} ; \mathbb{F}_{2}\right) \rightarrow H^{*}\left(S^{\infty} \times_{\Sigma_{2}}\right.$ $\left.M^{2} ; \mathbb{F}_{2}\right)$ satisfies $\mathrm{Sq}^{i}(n) \in N$ for $n \in N$. Thus, $\mathrm{Sq}^{i}(n)$ can be computed directly in $H^{*}\left(M^{2} ; \mathbb{F}_{2}\right)$ - with the Cartan formula. On the other hand, according to [3, Lemma 11] (see also [33, Section VI]), an element $a \otimes a \in D$ has $\operatorname{Sq}^{1}(a \otimes a)=$ $\left(\mathrm{Sq}^{1} a\right) \otimes a+a \otimes\left(\mathrm{Sq}^{1} a\right)$ only when $\operatorname{deg}(a)$ is even; otherwise $\mathrm{Sq}^{1}(a \otimes a)=z \otimes a \otimes$ $a+\left(\mathrm{Sq}^{1} a\right) \otimes a+a \otimes\left(\mathrm{Sq}^{1} a\right)$.

A simple diagram chase gives:

Corollary 8.3. Let $a \in H^{*}\left(S^{\infty} \times_{\Sigma_{2}} M^{2} ; \mathbb{F}_{2}\right)$. The following conditions are equivalent:

1. $j^{*}(a)=0$.

2. $\Delta^{*}(a)=\varphi(b)$ and $r_{2}(a)=\Delta_{\text {! }} \circ r_{1}(b)$, for some $b \in H^{*-m}\left(\mathrm{P}^{\infty} \times M ; \mathbb{F}_{2}\right)$.

Since $\varphi$ is monic, the term $b$ in Corollary 8.3 is unique; it is in fact the preimage of $a$ under the monomorphism $\mu: H^{*-m}\left(\mathrm{P}^{\infty} \times M ; \mathbb{F}_{2}\right) \rightarrow H^{*}\left(S^{\infty} \times_{\Sigma_{2}} M^{2} ; \mathbb{F}_{2}\right)$, whose complete image is of course the required ker $j^{*}$.

Remark 8.4. For calculations it is convenient to observe that $\mu$ is a map of $\mathbb{F}_{2}[z]$ modules; this follows from the behavior of $\Delta^{*}$, and the described multiplicative structure in (17).

Thus, concrete numerical calculations for the ring structure in $H^{*}\left(B(M, 2) ; \mathbb{F}_{2}\right)$ require knowledge of the Stiefel-Whitney classes $w_{i}$, the action of the Steenrod algebra on $M$, and the diagonal class $\delta$ associated to $M$. For the latter, it is convenient to keep in mind the following characterization.

Theorem 8.5 (Theorem 11.11 in $[23]$ ). Fix a basis $b_{1}, \ldots, b_{r}$ of $H^{*}\left(M ; \mathbb{F}_{2}\right)$, and let $b_{1}^{\prime}, \ldots, b_{r}^{\prime}$ stand for the corresponding dual basis. Then $\delta=\sum_{i=1}^{r} b_{i} \otimes b_{i}^{\prime}$.

Next, Haefliger's analysis will be specialized to the case $M=\mathrm{P}^{m}$ (the author does not know if this has been done elsewhere; however, the calculation implicit 
from [21, Theorem 3.7] should be noted). Start from (17) by noticing that $D=$ $\mathbb{F}_{2}[\lambda] / \lambda^{m+1}$, where $\lambda=z \otimes z$. Here $z$ stands for the restriction to $\mathrm{P}^{m}$ of the generator $z \in H^{1}\left(\mathrm{P}^{\infty} ; \mathbb{F}_{2}\right)$. The reader should keep in mind that this notation has a different use than that intended in (17), but the context clarifies any possible confusion. For instance, an additive $\mathbb{F}_{2}$-basis for $N$ is given by the monomials

$$
z^{i} \otimes z^{k}+z^{k} \otimes z^{i}, \quad 0 \leq i<k \leq m,
$$

whereas an $\mathbb{F}_{2}$-basis for the first summand on the right-hand side of (17) is given by the elements $z^{i} \otimes \lambda^{j}=z^{i} \otimes z^{j} \otimes z^{j}$, with $i, j \geq 0$ and $j \leq m$.

Theorem 8.6. Under the notation above, set $\zeta=z \otimes 1 \otimes 1, \eta=1 \otimes z+z \otimes 1$, and (as done above) $\lambda=z \otimes z$. Consider the polynomial expressions $Q_{i}=Q_{i}(\lambda, \eta) \in N$ defined by the relations $\eta^{i}+Q_{i}=1 \otimes z^{i}+z^{i} \otimes 1$ for $i \geq 1$, and set $Q_{0}=0$. Then there is a ring isomorphism

$$
H^{*}\left(B\left(\mathrm{P}^{m}, 2\right) ; \mathbb{F}_{2}\right) \approx \mathbb{F}_{2}[\zeta, \lambda, \eta] / I_{m},
$$

where $I_{m}$ is the ideal generated by $\zeta \eta$, the elements $R_{i}=\lambda^{m+1-i}\left(\eta^{i}+Q_{i}\right)$ for $0 \leq i \leq m+1$, and the elements $\mu\left(1 \otimes z^{k}\right)$ for $0 \leq k \leq m$, where $\mu$ is the map in Theorem 8.1 .

Proof. Note that the basis in (22) takes the form $\lambda^{i}\left(\eta^{k-i}+Q_{k-i}\right)$. An upper triangular matrix then changes (22) to the basis $\lambda^{i} \eta^{k-i}$ for $N$. This shows that

$$
H^{*}\left(S^{\infty} \times_{\Sigma_{2}}\left(\mathrm{P}^{m}\right)^{2} ; \mathbb{F}_{2}\right) \approx \mathbb{F}_{2}[\zeta, \lambda, \eta] /\left(\zeta \eta, R_{0}, \ldots, R_{m+1}\right) .
$$

Theorem 8.1 and Remark 8.4 then yield (23).

Remark 8.7. The given set of generators for $I_{m}$ is not minimal: take $m=2$; then (28)-(30) below show that $\mu\left(1 \otimes z^{2}\right)$ and all the generators $R_{i}$ are redundant, so that $I_{2}=\left(\zeta \eta, \lambda+\zeta^{2}+\eta^{2}, \zeta \lambda+\eta \lambda\right)$. This leads to (31) below, after eliminating the variable $\lambda$.

In order to make (23) into an explicit expression, one would need to know the $Q_{i}$ and the $\mu\left(1 \otimes z^{k}\right)$ as polynomials in the variables $\zeta, \lambda, \eta$. The former set of polynomials depends only on $\lambda$ and $\eta$ and, in fact, the relations defining the elements $Q_{i}$ can be used to get the inductive formula

$$
Q_{i}=\sum_{k \geq 1}^{\left[\frac{i-1}{2}\right]}\left(\begin{array}{l}
i \\
k
\end{array}\right) \lambda^{k}\left(\eta^{i-2 k}+Q_{i-2 k}\right)
$$

Here $\left[\frac{i-1}{2}\right]$ stands for the integral part of $(i-1) / 2$. For instance: $Q_{0}=Q_{1}=$ $Q_{2}=Q_{4}=Q_{8}=0, Q_{3}=\lambda \eta, Q_{5}=\lambda \eta^{3}+\lambda^{2} \eta, Q_{6}=\lambda^{2} \eta^{2}$, and $Q_{7}=\lambda \eta^{5}+\lambda^{3} \eta$. As for the polynomials $\mu\left(1 \otimes z^{k}\right)$, Theorem 8.1 can be used, in principle, to get noninductive expressions for these elements as soon as one knows the three maps $\Delta_{!}, \Delta^{*}$, and $\varphi$ in the case $M=\mathrm{P}^{m}$ (expressions for $r_{1}$ and $r_{2}$ are simple; the latter, for instance, is the identity on $D$ and $N$, but vanishes on any $z$-multiple). $\Delta_{!}$is 
determined by (20) and Theorem 8.5 which yields

$$
\delta=z^{m} \otimes 1+z^{m-1} \otimes z+\cdots+z \otimes z^{m-1}+1 \otimes z^{m} .
$$

Expression (21) can be written in a very compact form: since $W_{\mathrm{P}^{m}}=(1+z)^{m+1}$ and multiplication by $z \otimes 1$ is injective in $H^{*}\left(\mathrm{P}^{\infty} \times \mathrm{P}^{m} ; \mathbb{F}_{2}\right), \varphi$ is multiplication by

$$
\frac{(1 \otimes z+z \otimes 1)^{m+1}}{z \otimes 1},
$$

which is well defined since $0=1 \otimes z^{m+1} \in H^{*}\left(\mathrm{P}^{\infty} \times \mathrm{P}^{m} ; \mathbb{F}_{2}\right)$. Likewise, (18) takes the compact form

$$
\Delta^{*}\left(z^{k} \otimes z^{k}\right)=\left(1 \otimes z^{k}\right)(1 \otimes z+z \otimes 1)^{k} .
$$

Although the above information suffices to perform explicit computations, details soon get combinatorially complex as $m$ increases. Thus, after the following technical lemma (needed in connection with Project 1), only a few complete examples will be analyzed (for $m \leq 3$ ). In addition, the final part of this section offers a description of the height of the first Stiefel-Whitney class of the bundle $\zeta_{m}$ in Section 3 for some families of $m$, and its relation, in terms of Project 1, to the lower bounds in Table 1 .

Lemma 8.8 (Compare to 3, page 278]). For a closed smooth m-dimensional manifold $M$, the classifying map $B(M, 2) \rightarrow \mathrm{P}^{\infty}$ for the principal $\Sigma_{2}$-bundle $F(M, 2) \rightarrow$ $B(M, 2)$ corresponds to the $\mathbb{F}_{2}$-cohomology class $\zeta=j^{*}(z \otimes 1 \otimes 1)$.

Proof. This follows from the commutative diagram

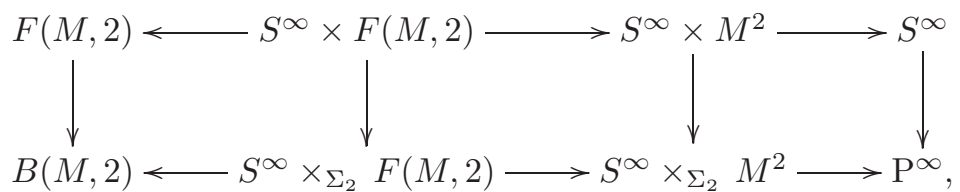

where the left-hand side horizontal maps are homotopy equivalences, the middle horizontal maps are inclusions, and the right-hand side square is a pull-back with horizontal maps projecting to the first coordinate.

Examples 8.9. Take $M=\mathrm{P}^{2}$, the projective plane, so that $W_{\mathrm{P}^{2}}=1+z+z^{2}$. Thus both (25) and (26) are given by $z^{2} \otimes 1+z \otimes z+1 \otimes z^{2}$. A direct calculation using (27) then shows that the $\mathbb{F}_{2}[z]$-monomorphism $\mu$ in Theorem 8.1 is determined by

$$
\begin{aligned}
& \mu(1 \otimes 1)=z^{2} \otimes 1 \otimes 1+1 \otimes z \otimes z+\left(z^{2} \otimes 1+1 \otimes z^{2}\right)=\zeta^{2}+\lambda+\eta^{2} \\
& \mu(1 \otimes z)=z \otimes z \otimes z+\left(z^{2} \otimes z+z \otimes z^{2}\right)=\zeta \lambda+\lambda \eta ; \\
& \mu\left(1 \otimes z^{2}\right)=1 \otimes z^{2} \otimes z^{2}=\lambda^{2}
\end{aligned}
$$

(observe that $\operatorname{dim}(\lambda)=2$ and $\operatorname{dim}(\zeta)=\operatorname{dim}(\eta)=1$ ) and, after a little algebraic manipulation, (23) becomes

$$
H^{*}\left(B\left(\mathrm{P}^{2}, 2\right) ; \mathbb{F}_{2}\right)=\mathbb{F}_{2}[\zeta, \eta] /\left(\zeta \eta, \zeta^{3}+\eta^{3}\right) .
$$

In a similar manner one derives

$$
H^{*}\left(B\left(\mathrm{P}^{3}, 2\right) ; \mathbb{F}_{2}\right)=\mathbb{F}_{2}[\zeta, \lambda, \eta] /\left(\zeta \eta, \lambda^{3}, \zeta^{3}+\eta^{3}, \zeta \lambda^{2}+\lambda^{2} \eta, \zeta^{2} \lambda+\lambda^{2}+\lambda \eta^{2}\right) .
$$

Table 2 gives an explicit additive $\mathbb{F}_{2}$-basis for this algebra, whereas Remark 8.2 gives the formulas $\mathrm{Sq}^{1}(\zeta)=\zeta^{2}, \mathrm{Sq}^{1}(\eta)=\eta^{2}$, and $\mathrm{Sq}^{1}(\lambda)=\lambda(\zeta+\eta)$ (the first two 
are forced by dimensional reasons). In Section 9 we will need to use some information about the $\mathrm{Sq}^{1}$-cohomology of $H^{*}\left(B\left(\mathrm{P}^{3}, 2\right) ; \mathbb{F}_{2}\right)$. A straightforward calculation shows this to be given by:

- $\mathbb{Z}_{2}$, in dimensions 0 and 4 (represented by 1 and $\lambda\left(\zeta^{2}+\eta^{2}\right)$, respectively);

- $\mathbb{Z}_{2} \oplus \mathbb{Z}_{2}$, in dimension 3 (represented by $\zeta^{3}$ and $\lambda \zeta$ );

- 0 , in any other dimension.

TABle 2. Basis elements in $H^{*}\left(B\left(\mathrm{P}^{3}, 2\right) ; \mathbb{F}_{2}\right)$

\begin{tabular}{|c|c|c|c|c|c|c|}
\hline basis & 1 & $\zeta, \eta$ & $\zeta^{2}, \eta^{2}, \lambda$ & $\zeta^{3}, \lambda \zeta, \lambda \eta$ & $\lambda \zeta^{2}, \lambda \eta^{2}$ & $\lambda \zeta^{3}$ \\
\hline dimension & 0 & 1 & 2 & 3 & 4 & 5 \\
\hline
\end{tabular}

Remark 8.10. The relations $\eta^{4}=0$ and $\zeta^{4}=0$ clearly hold in (31), but neither $\eta^{3}$ nor $\zeta^{3}$ vanishes. Of particular interest is the nontriviality of the last element since it implies, from Lemma 8.8, that the classifying map for $\zeta_{2}$ cannot be deformed into a map $B\left(\mathrm{P}^{2}, 2\right) \rightarrow \mathrm{P}^{2}$, and therefore, as described in Section $3 . \mathrm{TC}^{S}\left(\mathrm{P}^{2}\right) \geq 4$ (which is in fact an equality, as indicated in Table 1). This approach can be tried for larger-dimensional projective spaces (details below), but the lower bounds thus obtained do not improve on (but, for $m \leq 8$, coincide with) those in [15]. (The situation is comparable with that observed in the first complete paragraph on page 126 of 21.) For instance, although $\zeta^{4}=0$ is clearly a relation in $H^{*}\left(B\left(\mathrm{P}^{3}, 2\right) ; \mathbb{F}_{2}\right)$, $\zeta^{3}$ does not vanish in this ring (its restriction to $H^{*}\left(B\left(\mathrm{P}^{2}, 2\right) ; \mathbb{F}_{2}\right)$ is nontrivial). Thus $\mathrm{TC}^{S}\left(\mathrm{P}^{3}\right) \geq 4$ is all one can deduce from this $\mathbb{F}_{2}$-approach. But much of the motivation for Project 1 comes from the fact that, by replacing $\mathbb{F}_{2}$-cohomology with integral cohomology, the above ideas allow us to get, in Section 9 , the improved $\mathrm{TC}^{S}\left(\mathrm{P}^{3}\right) \geq 5$, a sharp inequality in view of Remark 2.2 and the known $\operatorname{Emb}\left(\mathrm{P}^{3}\right)=$ 5. (Remark 9.2 pinpoints the reason why the $\mathbb{F}_{2}$-approach fails.)

The rest of the section is devoted to describing the height of $\zeta \in H^{*}\left(B\left(\mathrm{P}^{m}, 2\right) ; \mathbb{F}_{2}\right)$ for some families of values of $m$, and to indicating the way this compares to the lower bounds in Table 1

Case $m=1$. One gets $R_{0}=\lambda^{2}, R_{1}=\lambda \eta, R_{2}=\eta^{2}, \mu(1 \otimes 1)=\zeta+\eta$, and $\mu(z \otimes z)=\lambda$. Thus $I_{1}$ reduces to the ideal generated by $\zeta \eta, \eta^{2}, \zeta+\eta$, and $\lambda$, and $H^{*}\left(B\left(\mathrm{P}^{1}, 2\right) ; \mathbb{F}_{2}\right) \approx \mathbb{F}_{2}[\zeta] / \zeta^{2}$, which, of course, is compatible with the fact that $B\left(\mathrm{P}^{1}, 2\right) \simeq S^{1}$. Under these conditions, the Hopf-type $\mathbb{F}_{2}$-approach in Section 3 gives $\operatorname{TC}^{S}\left(\mathrm{P}^{1}\right) \geq 2$, which is optimal in view of Table 1

Case $m=2^{e}$. As a partial generalization of the previous case, it is now affirmed that $0 \neq \zeta^{2^{e+1}-1} \in H^{*}\left(B\left(\mathrm{P}^{2^{e}}, 2\right) ; \mathbb{F}_{2}\right)$, so that the Hopf-type $\mathbb{F}_{2}$-approach in Section 3 gives $\mathrm{TC}^{S}\left(\mathrm{P}^{2^{e}}\right) \geq 2^{e+1}$, which is optimal for $e \leq 3$ in view of Table 1 and for $e \geq 4$ in view of [6, 15]. Indeed, in the notation of Theorem 8.1] one has $\Delta^{*}\left(z^{2^{e+1}-1} \otimes 1 \otimes\right.$ $1)=z^{2^{e+1}-1} \otimes 1$. But an easy calculation shows that the preimage of this element under $\varphi$ is

$$
z^{2^{e}-1} \otimes 1+z^{2^{e}-2} \otimes z+\cdots+z \otimes z^{2^{e}-2}+1 \otimes z^{2^{e}-1} .
$$

However, the last element maps nontrivially under $r_{1}$, while $r_{2}\left(z^{2^{e+1}-1} \otimes 1 \otimes 1\right)=0$. 
Case $m=2^{e}+\varepsilon$ with $\varepsilon \in\{1,2\}$ and $e \geq 2$. The previous analysis implies that $0 \neq \zeta^{2^{e+1}-1} \in H^{*}\left(B\left(\mathrm{P}^{2^{e}+\varepsilon}, 2\right) ; \mathbb{F}_{2}\right)$. It is now affirmed that $0=\zeta^{2^{e+1}} \in$ $H^{*}\left(B\left(\mathrm{P}^{2^{e}+\varepsilon}, 2\right) ; \mathbb{F}_{2}\right)$, so that the best information one gets from the Hopf-type $\mathbb{F}_{2^{-}}$ approach in Section $\left[3\right.$ is $\mathrm{TC}^{S}\left(\mathrm{P}^{2^{e}+\varepsilon}\right) \geq 2^{e+1}$. Indeed, it is enough to consider the case $\varepsilon=2$, where a new calculation gives that the $\varphi$-preimage of $\Delta^{*}\left(z^{2^{e+1}} \otimes 1 \otimes 1\right)=$ $z^{2^{e+1}} \otimes 1$ is

$$
\sum_{k \geq 0}^{2^{e-2}-1}\left(z^{2^{e}-4 k-2} \otimes z^{4 k}+z^{2^{e}-4 k-3} \otimes z^{4 k+1}\right) .
$$

The last element maps trivially under $r_{1}$, so that it is in fact the $\mu$-preimage of $z^{2^{e+1}} \otimes 1 \otimes 1$, killing $\zeta^{2^{e+1}} \in H^{*}\left(B\left(\mathrm{P}^{2^{e}+\varepsilon}, 2\right) ; \mathbb{F}_{2}\right)$.

Remark 8.11. Since $\mathrm{TC}^{S}\left(\mathrm{P}^{a}\right) \geq \mathrm{TC}^{S}\left(\mathrm{P}^{b}\right)$ holds for $a \geq b$ (recall Theorem 2.1), the inequality $\mathrm{TC}^{S}\left(\mathrm{P}^{2^{e}+\varepsilon}\right) \geq 2^{e+1}$ (for $\varepsilon \in\{1,2\}$ ) follows directly from the previously established $\mathrm{TC}^{S}\left(\mathrm{P}^{2^{e}}\right) \geq 2^{e+1}$. But unlike the latter, the former is actually not optimal for $e \geq 3$ : Table 1 gives $\operatorname{TC}^{S}\left(\mathrm{P}^{9}\right)=\mathrm{TC}^{S}\left(\mathrm{P}^{10}\right)=17$, whereas $\mathrm{TC}^{S}\left(\mathrm{P}^{2^{e}+\varepsilon}\right)=2^{e+1}+1$ for $e \geq 4$ in view of 6, 15. In view of its success for $(e, \varepsilon)=(1,1)$, there seems to be a "good" chance that the ideas in Project 1 can be used to settle the still unresolved cases with $e=2(\varepsilon=1,2)$. Of course, this would settle the value of $\operatorname{TC}^{S}\left(\mathrm{P}^{m}\right)$ for $m \leq 6$ and, on the other hand, it would show the equality $\mathrm{TC}^{S}\left(\mathrm{P}^{m}\right)=\operatorname{Emb}\left(\mathrm{P}^{m}\right)$ for $m \leq 5$.

Case $m=2^{e}+3$. Just as in previous situations,

$$
0=\zeta^{2^{e+1}} \in H^{*}\left(B\left(\mathrm{P}^{2^{e}+3}, 2\right) ; \mathbb{F}_{2}\right),
$$

so that the best information one gets from the Hopf-type $\mathbb{F}_{2}$-approach in Section 3 is again $\mathrm{TC}^{S}\left(\mathrm{P}^{2^{e}+3}\right) \geq 2^{e+1}$; besides having one further illustration of Haefliger's method, the reason for not including this case with the previous one is that there is currently no clear evidence as to what the actual value of $\mathrm{TC}^{S}\left(\mathrm{P}^{2^{e}+3}\right)$ could be. To show (32), this time one computes that the $\varphi$-preimage of $\Delta^{*}\left(z^{2^{e+1}} \otimes 1 \otimes 1\right)=$ $z^{2^{e+1}} \otimes 1$ is

$$
\sum_{k \geq 0}^{2^{e-2}-1}\left(z^{2^{e}-4 k-3} \otimes z^{4 k}\right) .
$$

The last element maps trivially under $r_{1}$, so that it is in fact the $\mu$-preimage of $z^{2^{e+1}} \otimes 1 \otimes 1$, once again killing $\zeta^{2^{e+1}} \in H^{*}\left(B\left(\mathrm{P}^{2^{e}+3}, 2\right) ; \mathbb{F}_{2}\right)$.

\section{Proofs of Theorems 1.4 and 1.5}

Unless otherwise noted, throughout this section $H^{*}(X)$ stands for the singular cohomology groups (or algebra, depending on the context) of a space $X$, where integral coefficients are used.

It has been observed, at the end of Remark 8.10, that in order to settle the equality $\mathrm{TC}^{S}\left(\mathrm{P}^{3}\right)=5$, it is enough to prove $\mathrm{TC}^{S}\left(\mathrm{P}^{3}\right) \geq 5$. This inequality is established in the present section within the setup in Project 1 Indeed, as explained in Section 3 , the goal is to show that the classifying map for $\zeta_{3}$, i.e., the line bundle associated to the canonical $\Sigma_{2}$-covering projection $F\left(\mathrm{P}^{3}, 2\right) \rightarrow B\left(\mathrm{P}^{3}, 2\right)$, cannot be compressed into a map

$$
B\left(\mathrm{P}^{3}, 2\right) \rightarrow \mathrm{P}^{3}
$$


Since $H^{*}\left(\mathrm{P}^{\infty}\right)=\mathbb{Z}[\omega] /(2 \omega), \omega \in H^{2}\left(\mathrm{P}^{\infty}\right)$, with $\omega^{2}$ trivial on $\mathrm{P}^{3}$, the required conclusion can be stated as:

Theorem 9.1. $\omega^{2}$ maps nontrivially under the classifying map for $\zeta_{3}$.

Remark 9.2. Let $\widehat{\zeta_{3}}: B\left(\mathrm{P}^{3}, 2\right) \rightarrow \mathrm{P}^{\infty}$ stand for the map in Theorem 9.1 It will become clear that $\widehat{\zeta_{3}^{*}}\left(\omega^{2}\right)$ is divisible by 2 . This is the reason why cohomology with mod 2 coefficients is not useful for proving the required inequality $\mathrm{TC}^{S}\left(\mathrm{P}^{3}\right) \geq 5$.

The proof of Theorem 9.1 is based on the Cartan-Leray-Serre spectral sequence (with integral coefficients) for the $\Sigma_{2}$-cover associated to $\zeta_{3}$. Much of this section is devoted to giving full details of that spectral sequence.

Recall that the homotopy fiber of $\widehat{\zeta}_{3}$ is the ordered configuration space $F\left(\mathrm{P}^{3}, 2\right)$ with $\left(\Sigma_{2}=\pi_{1}\left(\mathrm{P}^{\infty}\right)\right)$-action given by the involution $(u, v) \stackrel{t}{\mapsto}(v, u)$. Thus, the spectral sequence to be used has

$$
E_{2}^{p, q}=H^{p}\left(\mathrm{P}^{\infty} ; H^{q}\left(F\left(\mathrm{P}^{3}, 2\right)\right)\right) \Longrightarrow H^{p+q}\left(B\left(\mathrm{P}^{3}, 2\right)\right) .
$$

Cohomology coefficients in this $E_{2}$-term are twisted by (the map induced by) $t$. A sound hold on (33) comes from the homeomorphism $\mathrm{P}^{3} \times\left(\mathrm{P}^{3}-\{e\}\right) \cong F\left(\mathrm{P}^{3}, 2\right)$ given by $(a, b) \mapsto(a, a b)$ with inverse $(x, y) \mapsto\left(x, x^{-1} y\right)$, where $e$ is the identity matrix in $\mathrm{P}^{3}=\mathrm{SO}(3)$, and inverses are taken with respect to the group structure. In these terms, the resulting involution $\tau: \mathrm{P}^{3} \times\left(\mathrm{P}^{3}-\{e\}\right) \rightarrow \mathrm{P}^{3} \times\left(\mathrm{P}^{3}-\{e\}\right)$ takes the form $\tau(a, b)=\left(a b, b^{-1}\right)$.

Recall $H^{*}\left(\mathrm{P}^{3}\right)=\mathbb{Z}[x, y] /\left(x^{2}, y^{2}, x y, 2 x\right)$ and $H^{*}\left(\mathrm{P}^{3}-\{e\}\right)=\mathbb{Z}[x] /\left(x^{2}, 2 x\right)$, for cohomology classes $x$ and $y$ of dimensions 2 and 3, respectively. The Künneth isomorphism $H^{*}(X \times Y) \approx\left[H^{*}(X) \otimes H^{*}(Y)\right]^{*} \oplus\left[\operatorname{Tor}\left(H^{*}(X), H^{*}(Y)\right)\right]^{*+1}$ yields:

Lemma 9.3. $H^{*}\left(\mathrm{P}^{3} \times\left(\mathrm{P}^{3}-\{e\}\right)\right)$ is the direct sum of

$$
\mathbb{Z}\left[x_{1}, y_{1}, x_{2}\right] /\left(x_{1}^{2}, x_{2}^{2}, y_{1}^{2}, x_{1} y_{1}, 2 x_{1}, 2 x_{2}\right)
$$

and a copy of $\mathbb{Z} / 2$ generated by a class $z \in H^{3}\left(\mathrm{P}^{3} \times\left(\mathrm{P}^{3}-\{e\}\right)\right)$.

The index $i$ in $x_{i}$ and $y_{i}$ refers to the Cartesian factor where the indicated classes originate. The only class coming from the Tor part, the class $z$, arises from the two groups $H^{2}\left(\mathrm{P}^{3}\right) \approx H^{2}\left(\mathrm{P}^{3}-\{e\}\right) \approx \mathbb{Z} / 2$. The whole multiplicative structure in $H^{*}\left(\mathrm{P}^{3} \times\left(\mathrm{P}^{3}-\{e\}\right)\right)$ is determined by specifying the four products $x_{1} z, x_{2} z$, $y_{1} z$, and $z^{2}$. Of these, the last two are trivial for dimensional reasons, whereas Example 9.5 below settles the corresponding fact for $x_{2} z$. Although irrelevant for the calculations in this section, the author does not know whether $x_{1} z$ is trivial or not $\left(x_{1} z=y_{1} x_{2}\right.$ would be forced in the latter case).

The next step toward understanding (33) is to produce a complete description of $\tau^{*}: H^{*}\left(\mathrm{P}^{3} \times\left(\mathrm{P}^{3}-\{e\}\right)\right) \rightarrow H^{*}\left(\mathrm{P}^{3} \times\left(\mathrm{P}^{3}-\{e\}\right)\right)$. This involution is easily seen to be trivial in the cases $H^{0}\left(\mathrm{P}^{3} \times\left(\mathrm{P}^{3}-\{e\}\right)\right)=\mathbb{Z}, H^{1}\left(\mathrm{P}^{3} \times\left(\mathrm{P}^{3}-\{e\}\right)\right)=0$, $H^{4}\left(\mathrm{P}^{3} \times\left(\mathrm{P}^{3}-\{e\}\right)\right) \approx \mathbb{Z} / 2$, and $H^{5}\left(\mathrm{P}^{3} \times\left(\mathrm{P}^{3}-\{e\}\right)\right) \approx \mathbb{Z} / 2$. The following result gives the answer in the two remaining cases.

Proposition 9.4. In the cohomology of dimensions 2 and 3 , the involution $\tau^{*}$ satisfies

$$
\tau^{*}\left(x_{1}\right)=x_{1}+x_{2}, \quad \tau^{*}\left(x_{2}\right)=x_{2}, \quad \tau^{*}\left(y_{1}\right)=y_{1}+z, \quad \text { and } \quad \tau^{*}(z)=z .
$$

Example 9.5. The relation $x_{2} z=0$ follows from $x_{1} z=\tau^{*}\left(x_{1} z\right)=\tau^{*}\left(x_{1}\right) \tau^{*}(z)=$ $\left(x_{1}+x_{2}\right) z=x_{1} z+x_{2} z$. 
Proof. The formula $\tau^{*}(z)=z$ follows from the observation that $z$ is the unique element in $H^{3}\left(\mathrm{P}^{3} \times\left(\mathrm{P}^{3}-\{e\}\right)\right) \approx \mathbb{Z} \oplus \mathbb{Z} / 2$ of order two. For the remaining cases, consider the diagram

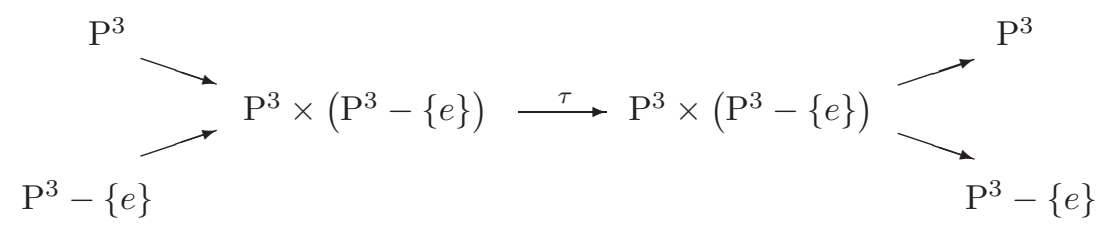

where right-hand side diagonal maps are Cartesian projections, and left-hand side diagonal maps are Cartesian inclusions with respect to some chosen base point $d \in \mathrm{P}^{3}-\{e\} \subset \mathrm{P}^{3}=\mathrm{SO}(3)$ of order two (e.g., $d=\operatorname{Diag}(-1,-1,1)$, so that $\left.d=d^{-1}\right)$. The four resulting components are depicted in
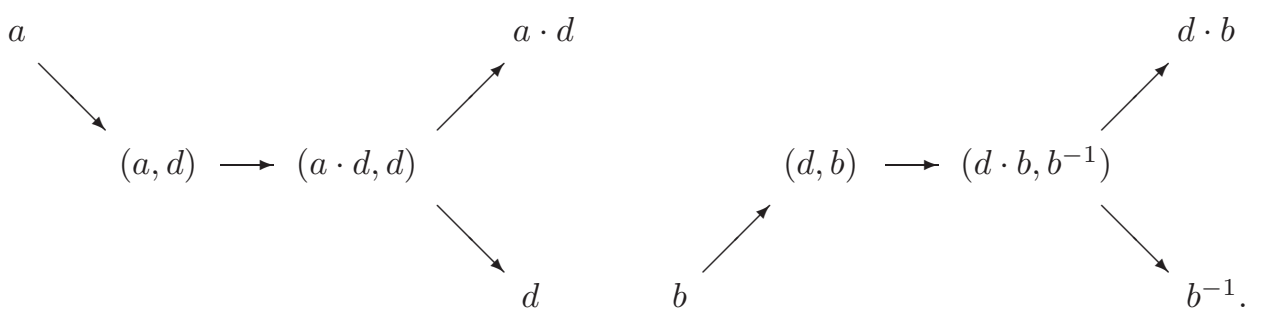

Since $\mathrm{P}^{3}$ is a path-connected group, the components $\mathrm{P}^{3} \rightarrow \mathrm{P}^{3}$ and $\mathrm{P}^{3}-\{e\} \rightarrow \mathrm{P}^{3}$ are homotopic to inclusions, whereas the component $\mathrm{P}^{3}-\{e\} \rightarrow \mathrm{P}^{3}-\{e\}$ is necessarily the identity in $H^{2}\left(\mathrm{P}^{3}-\{e\}\right) \approx \mathbb{Z} / 2$. This yields the first two formulas in (34). However, since $z$ is not detected by axial inclusions, all one gets for the third formula in (34) is $\tau^{*}\left(y_{1}\right)=y_{1}+\epsilon z$, for some $\epsilon \in\{0,1\}$. In order to settle this indeterminacy, consider the portion

$$
\begin{aligned}
H^{3}\left(\mathrm{P}^{3} \times\left(\mathrm{P}^{3}-\{e\}\right)\right) & \stackrel{2}{\rightarrow} H^{3}\left(\mathrm{P}^{3} \times\left(\mathrm{P}^{3}-\{e\}\right)\right) \stackrel{\text { proj }}{\rightarrow} H^{3}\left(\mathrm{P}^{3} \times\left(\mathrm{P}^{3}-\{e\}\right) ; \mathbb{Z} / 2\right) \\
(35) & \stackrel{\partial}{\rightarrow} H^{4}\left(\mathrm{P}^{3} \times\left(\mathrm{P}^{3}-\{e\}\right)\right) \stackrel{2}{\rightarrow} H^{4}\left(\mathrm{P}^{3} \times\left(\mathrm{P}^{3}-\{e\}\right)\right)
\end{aligned}
$$

of the long exact sequence associated to the extension $0 \rightarrow \mathbb{Z} \stackrel{2}{\rightarrow} \mathbb{Z} \stackrel{\text { proj }}{\rightarrow} \mathbb{Z} / 2 \rightarrow 0$. Since $H^{3}\left(\mathrm{P}^{3} \times\left(\mathrm{P}^{3}-\{e\}\right)\right) \approx \mathbb{Z} \oplus \mathbb{Z} / 2$ and $H^{4}\left(\mathrm{P}^{3} \times\left(\mathrm{P}^{3}-\{e\}\right)\right) \approx \mathbb{Z} / 2$, the middle part in (35) becomes

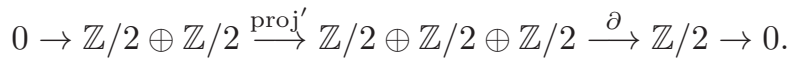

Thus, the value of $\epsilon$ can be determined by looking at $\left.\tau^{*}: H^{3}\left(\mathrm{P}^{3}-\{e\}\right) ; \mathbb{Z} / 2\right)$ $\rightarrow H^{3}\left(\mathrm{P}^{3} \times\left(\mathrm{P}^{3}-\{e\}\right) ; \mathbb{Z} / 2\right)$. Indeed, $\epsilon=1$ is forced from the commutative diagram

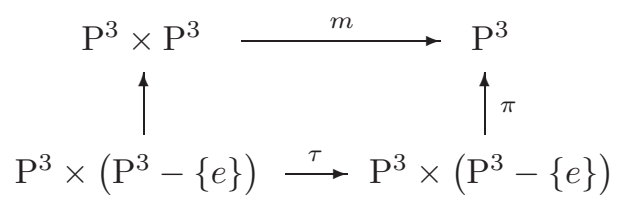


(where the vertical map on the left-hand side is the obvious inclusion, and $m$ is the multiplication in $\left.\mathrm{P}^{3}=\mathrm{SO}(3)\right)$ and the well-known fact that $m^{*}\left(g^{3}\right)=g^{3} \otimes 1+g^{2} \otimes$ $g+g \otimes g^{2}+1 \otimes g^{3}$, where $g$ is the generator in $H^{1}\left(\mathrm{P}^{3} ; \mathbb{Z} / 2\right)$.

The $E_{2}$-term in (33) can now be obtained from standard calculations (e.g. 7, page 6]). The result, recorded next, is depicted in the chart following Remark 9.7 .

Corollary 9.6. 1. $H^{*}\left(\mathrm{P}^{\infty} ; H^{0}\left(\mathrm{P}^{3} \times\left(\mathrm{P}^{3}-\{e\}\right)\right)\right)=\mathbb{Z}[\omega] /(2 \omega), \operatorname{dim}(\omega)=2$.

2. $H^{*}\left(\mathrm{P}^{\infty} ; H^{1}\left(\mathrm{P}^{3} \times\left(\mathrm{P}^{3}-\{e\}\right)\right)\right)=0$.

3. $H^{*}\left(\mathrm{P}^{\infty} ; H^{2}\left(\mathrm{P}^{3} \times\left(\mathrm{P}^{3}-\{e\}\right)\right)\right)= \begin{cases}\mathbb{Z} / 2, & *=0 \\ 0, & *>0 .\end{cases}$

4. $H^{*}\left(\mathrm{P}^{\infty} ; H^{3}\left(\mathrm{P}^{3} \times\left(\mathrm{P}^{3}-\{e\}\right)\right)\right)= \begin{cases}\mathbb{Z} \oplus \mathbb{Z} / 2, & *=0 ; \\ \mathbb{Z} / 2, & \text { positive even } * ; \\ 0, & \text { odd } * .\end{cases}$

5. $H^{*}\left(\mathrm{P}^{\infty} ; H^{q}\left(\mathrm{P}^{3} \times\left(\mathrm{P}^{3}-\{e\}\right)\right)\right)=\mathbb{F}_{2}\left[\omega_{q}\right], \quad \operatorname{dim}\left(\omega_{q}\right)=1, \quad q=4,5$.

6. $H^{*}\left(\mathrm{P}^{\infty} ; H^{i}\left(\mathrm{P}^{3} \times\left(\mathrm{P}^{3}-\{e\}\right)\right)\right)=0, \quad i \geq 6$.

Remark 9.7. An explicit description of the $H^{*}\left(\mathrm{P}^{\infty}\right)$-module structure in the spectral sequence (33) will be crucial for getting a good control of differentials (in the next paragraphs). To begin with, as indicated in Corollary 9.6(1), there is the standard copy of the ring $H^{*}\left(\mathrm{P}^{\infty}\right)$ at the $(q=0)$-line of (33), whereas Corollary 9.6) (3) forces the $H^{*}\left(\mathrm{P}^{\infty}\right)$-module $H^{*}\left(\mathrm{P}^{\infty} ; H^{2}\left(\mathrm{P}^{3} \times\left(\mathrm{P}^{3}-\{e\}\right)\right)\right)$ at the $(q=2)$-line to have one generator (of dimension $p=0$ ) with both 2 and $\omega$ acting trivially. The situation at the lines $q=4$ and $q=5$ is well known; in the notation of Corollary 9.6 (5), the $H^{*}\left(\mathrm{P}^{\infty}\right)$-module $H^{*}\left(\mathrm{P}^{\infty} ; H^{q}\left(\mathrm{P}^{3} \times\left(\mathrm{P}^{3}-\{e\}\right)\right)\right)$ is generated by $1_{q}$ and $\omega_{q}$ subject to the single relation $2 \cdot 1_{q}=0$ (here $1_{q}$ stands for the unit of the ring in Corollary 9.6(5)). Finally, in order to interpret Corollary 9.6(4), note first that the last relation in (34) claims that $\mathbb{Z} / 2$ is a $\mathbb{Z}[\mathbb{Z} / 2]$-submodule of $H^{3}\left(\mathrm{P}^{3} \times\left(\mathrm{P}^{3}-\{e\}\right)\right)=\mathbb{Z} \oplus \mathbb{Z} / 2$. Then, a standard calculation shows that the induced map

$$
H^{*}\left(\mathrm{P}^{\infty} ; \mathbb{Z} / 2\right) \rightarrow H^{*}\left(\mathrm{P}^{\infty} ; H^{3}\left(\mathrm{P}^{3} \times\left(\mathrm{P}^{3}-\{e\}\right)\right)\right)
$$

is injective in even dimensions (and, therefore, an isomorphism in positive even dimensions). Thus, the commutative diagram

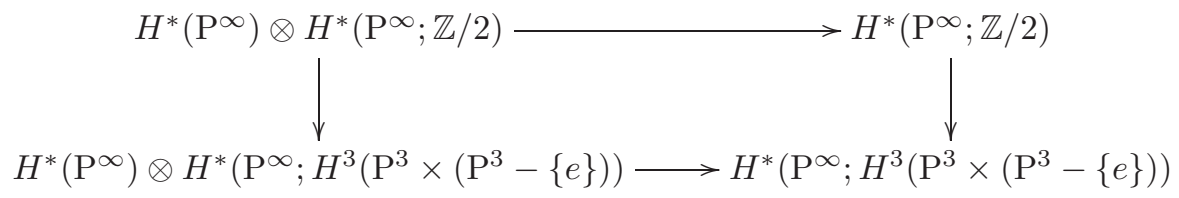

of $H^{*}\left(\mathrm{P}^{\infty}\right)$-actions implies that multiplication by $\omega \in H^{2}\left(\mathrm{P}^{\infty}\right)$ is monic on the 2-torsion part of $H^{*}\left(\mathrm{P}^{\infty} ; H^{3}\left(\mathrm{P}^{3} \times\left(\mathrm{P}^{3}-\{e\}\right)\right)\right.$. 
Here is a chart of the $E_{2}$-term in (33):

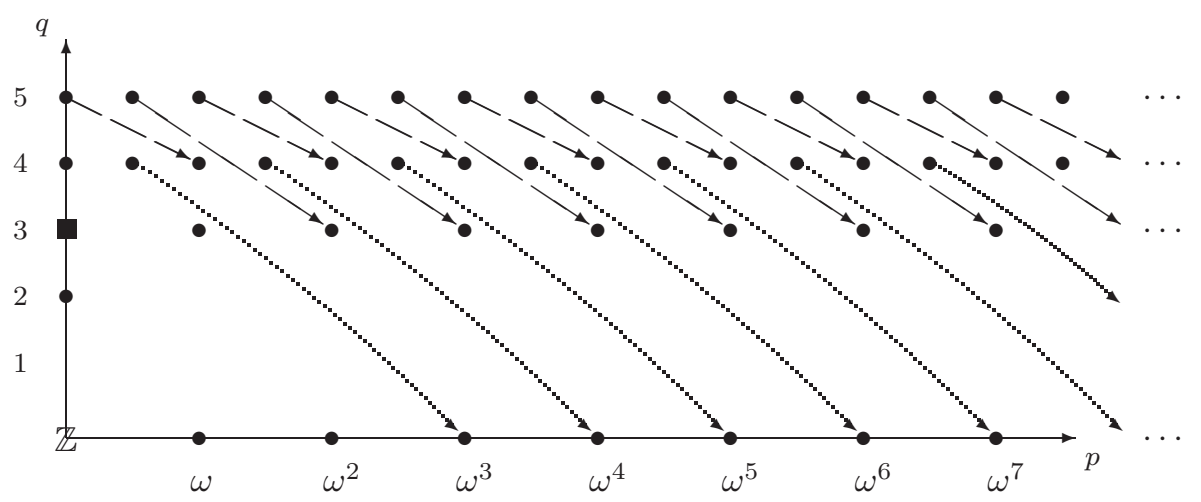

A bullet represents a copy of $\mathbb{Z} / 2$, and the solid square in $E_{2}^{0,3}$ stands for $\mathbb{Z} \oplus \mathbb{Z} / 2$. Note the group $\mathbb{Z}$ at node $(0,0)$. The dashed and dotted arrows are to be ignored for now; they will be shown to give the pattern of differentials in the spectral sequence.

One immediately sees from the chart that the first group in the short exact sequence (analogous to (35))

$$
H^{1}\left(B\left(\mathrm{P}^{3}, 2\right)\right) \rightarrow H^{1}\left(B\left(\mathrm{P}^{3}, 2\right) ; \mathbb{Z} / 2\right) \rightarrow H^{2}\left(B\left(\mathrm{P}^{3}, 2\right)\right) \stackrel{2}{\rightarrow} H^{2}\left(B\left(\mathrm{P}^{3}, 2\right)\right)
$$

is trivial. Coupled with the fact (coming from Table 2) that the second group in (36) is $\mathbb{Z} / 2 \oplus \mathbb{Z} / 2$, this implies that the element at node $(0,2)$ is a permanent cycle (this much is obvious since there is no possible nontrivial target for it) and that $H^{2}\left(B\left(\mathrm{P}^{3}, 2\right)\right)=\mathbb{Z} / 2 \oplus \mathbb{Z} / 2$, a trivial extension in this part of the $E_{\infty}$-term.

Next observe that the only potentially nontrivial differentials $d_{r}$ originating at node $(0,3)$ land at node $(4,0)-d_{4}$-differentials possibly hitting $\omega^{2}$. The kernel of such a differential is $H^{3}\left(B\left(\mathrm{P}^{3}, 2\right)\right)$, whose only possibilities are:

(i) $\mathbb{Z} \oplus \mathbb{Z} / 2$, if the torsion element at node $(0,3)$ is a $d_{4}$-cycle (independently of the $d_{4}$-image of the torsion-free element at node $\left.(0,3)\right)$;

(ii) $\mathbb{Z}$, if the torsion element at node $(0,3)$ hits, under $d_{4}$, the element at node $(4,0)$ (once again, independently of the $d_{4}$-image of the torsion-free element at node $(0,3))$.

Either one of these two possible cases gives at most one nontrivial $\mathrm{Sq}^{1}$-cohomology class in $H^{3}\left(B\left(\mathrm{P}^{3}, 2\right) ; \mathbb{Z} / 2\right)$, namely the one coming from the integral class. But the calculation at the end of Examples 8.9 shows that the $\mathrm{Sq}^{1}$-cohomology has rank 2 in dimension 3 , so that $H^{4}\left(B\left(\mathrm{P}^{3}, 2\right)\right)$ must have an element of order 4 . Therefore both elements in total degree 4 in the chart must survive to nontrivial classes in $E_{\infty}$ making up a nontrivial extension in $H^{4}\left(B\left(\mathrm{P}^{3}, 2\right)\right)=\mathbb{Z} / 4$. A number of consequences follow at this point:

(a) All elements at nodes $(0,3)$ and $(0,4)$ are permanent cycles.

(b) Possibility (i) above holds, that is, $H^{3}\left(B\left(\mathrm{P}^{3}, 2\right)\right)=\mathbb{Z} \oplus \mathbb{Z} / 2$.

(c) $\omega^{2}$ is the nontrivial element of order 2 in $H^{4}\left(B\left(\mathrm{P}^{3}, 2\right)\right)=\mathbb{Z} / 4$, proving Theorem 9.1 and the second part in Theorem 1.5 .

The only task remaining in the proof of Theorem 1.5 is the determination of $H^{5}\left(B\left(\mathrm{P}^{3}, 2\right)\right)$. This can be obtained in terms of the nonorientability (proved in [16]) of the 5-manifold mentioned in (111) as having the homotopy type of $B\left(\mathrm{P}^{3}, 2\right)$ or, 
alternatively, by using [3. Theorem 43]. Instead, having come this far, we finish up the description of the spectral sequence (33).

Together with the $H^{*}\left(\mathrm{P}^{\infty}\right)$-module structure described in Remark 9.7 (a) above implies that all elements in the $(q=3)$-line, as well as all elements at nodes $(2 i, 4)$ for $i \geq 0$, are permanent cycles. But the element at node $(2,4)$ cannot survive to a nontrivial class in $E_{\infty}$ (in view of (110), and this forces a nontrivial $d_{2}$-differential from node $(0,5)$ to node $(2,4)$. In fact, the $H^{*}\left(\mathrm{P}^{\infty}\right)$-module structure implies that all differentials $d_{2}: E_{2}^{2 i, 5} \rightarrow E_{2}^{2 i+2,4}$ are isomorphisms for $i \geq 0$, the family of $d_{2^{-}}$ differentials depicted in the chart above. Furthermore, the only other potentially nontrivial $d_{2}$-differentials are those of the form

$$
d_{2}^{2 i+1,5}: E_{2}^{2 i+1,5} \rightarrow E_{2}^{2 i+3,4}
$$

for $i \geq 0$. But if $d_{2}^{2 i+1,5}$ was nontrivial, then there would not be any class left to kill the permanent cycle at node $(2 i+4,3)$, which must be killed in view of (111). Therefore, all differentials in (37) are actually trivial and, instead, all differentials $d_{3}^{2 i+1,5}: E_{2}^{2 i+1,5} \rightarrow E_{2}^{2 i+4,3}(i \geq 0)$ are isomorphisms, giving the family of $d_{3}$-differentials depicted in the chart above (these, by the way, are compatible with the $H^{*}\left(\mathrm{P}^{\infty}\right)$-module structure). This accounts for all the possible nontrivial differentials up to $E_{5}$ (recall that every element in the $(q=3)$-line is a permanent cycle). Finally, the family of $d_{5}$-differentials depicted in the chart above is forced since classes $\omega^{i}$ with $i \geq 3$ must be killed in view of (11) (once again, the resulting family of $d_{5}$-differentials is compatible with the $H^{*}\left(\mathrm{P}^{\infty}\right)$-module structure). In particular, in total degrees 5 there is only one remaining $\mathbb{Z} / 2$, which makes up $H^{5}\left(B\left(\mathrm{P}^{3}, 2\right)\right)$.

Remark 9.8. Some of the properties in the multiplicative structure of the ring $H^{*}\left(B\left(\mathrm{P}^{3}, 2\right)\right)$ can be recovered from the analysis above. To begin with, we know that $\omega \in H^{2}\left(B\left(\mathrm{P}^{3}, 2\right)\right)$ has $\omega^{2} \neq 0$ but $\omega^{3}=0$. Next, it is asserted that there is a nontrivial exterior element $a \in H^{2}\left(B\left(\mathrm{P}^{3}, 2\right)\right.$ ) (so that $a$ and $\omega$ form a $\mathbb{Z} / 2$-basis of $\left.H^{2}\left(B\left(\mathrm{P}^{3}, 2\right)\right)\right)$. Indeed, pick any $a \notin\{0, \omega\}$; if $a^{2} \neq 0$, then $a^{2}=\omega^{2}$ would be forced, so that $(a+\omega)^{2}=0$. Unfortunately, once an exterior $a$ has been fixed, the author does not know how to decide whether $\omega a=0$ or $\omega a=\omega^{2}$ (both possibilities are compatible with the multiplicative structure seen from the spectral sequence). Further, there are the three indecomposable elements:

- $b \in H^{3}\left(B\left(\mathrm{P}^{3}, 2\right)\right)$, generating a $\mathbb{Z}$;

- $c \in H^{3}\left(B\left(\mathrm{P}^{3}, 2\right)\right)$, generating a $\mathbb{Z} / 2$;

- $d \in H^{4}\left(B\left(\mathrm{P}^{3}, 2\right)\right)$, generating a $\mathbb{Z} / 4$.

The relation $\omega^{2}=2 d$ has already been discussed, but now the fact that $\omega c$ is the generator of $H^{5}\left(B\left(\mathrm{P}^{3}, 2\right)\right)=\mathbb{Z} / 2$ follows from the multiplicative structure in the spectral sequence. As an exercise, the interested reader can check that the explicit formulas in [7, page 25] imply that, in the $H^{*}\left(\mathrm{P}^{\infty}\right)$-module structure discussed in Remark 9.7 multiplication by $\omega \in H^{*}\left(\mathrm{P}^{\infty}\right)$ sends the torsion-free generator in $H^{0}\left(\mathrm{P}^{\infty} ; H^{3}\left(\mathrm{P}^{3} \times\left(\mathrm{P}^{3}-\{e\}\right)\right)\right)$ into the generator in $H^{2}\left(\mathrm{P}^{\infty} ; H^{3}\left(\mathrm{P}^{3} \times\left(\mathrm{P}^{3}-\{e\}\right)\right)\right)$, so that $\omega b=\omega c$. The author does not know how to deal with the two remaining products $a b$ and $a c$ (as in the case of $\omega a$, their triviality in the spectral sequence just indicates that these products have filtration higher than expected). 


\section{Symmetric MOtion PlanNERS}

M. Farber began in [8, 9] a study of the continuity instabilities inherent in any motion planner for a robotical system. In this section, his methods and results are (partially) adapted to the symmetric case.

Suppose a given robotical system has to be programmed to perform tasks which, however, need to be decided during the course of the operation. Thus, the programming must be made in such a way that, after being fed with a given pair of states $(A, B)$ of the system, the robot should decide and perform, in an autonomous way, the required transformations for going from one of the given states to the other. There are three natural requirements that arise in many practical situations:

(1) Motion should be symmetric: the chosen movement from $A$ to $B$ should be the same one, but in the reverse direction, as the movement from $B$ to $A$.

(2) There is no need to plan motion from a given state to itself: the robot will only be fed with pairs of different states $(A \neq B)$.

(3) The programming should be robust enough to allow for small "errors" in the description of the states: if the states $A$ and $B$ change into slightly perturbed new states $A^{\prime}$ and $B^{\prime}$, then the corresponding movements $A \sim B$ and $A^{\prime} \sim B^{\prime}$ should be "roughly" the same.

Let $X$ be the space of all possible states the system can take. The topology of $X$ is determined by the capabilities and constraints of the system (e.g. physical design of the robot, or obstacles in the robot's path). Attention will be restricted to the case of a path-connected $X$, which means that the system can always be transformed from any given state to any other state. Then, the required programming is encoded by a continuous (so that condition 3 above holds) $\mathbb{Z} / 2$-equivariant (so that condition 1 above holds) section $s: F(X, 2) \rightarrow P_{1}(X)$ of the evaluation map ev ${ }_{1}: P_{1}(X) \rightarrow F(X, 2)$, where the diagonal has been removed from $X \times X$ to form $F(X, 2)$ in view of condition 2 above (see Section 1 for the relevant definitions about $\left.\mathrm{ev}_{1}\right)$. However, it is known that such a programming problem is solvable only in very special situations; indeed, the required section $s$ can exist only when $X$ has a two-sided unital homotopy comultiplication 6 (e.g. when $X$ is a suspension). Thus, the best one can hope for, in general, is to give a small number of continuous local moving instructions, that is, to be able to partition the space $F(X, 2)$ into a small number of pieces $F_{1}, \ldots, F_{k}$ each admitting a continuous $\mathbb{Z} / 2$-equivariant local section $s_{i}: F_{i} \rightarrow P_{1}(X)$ of $\mathrm{ev}_{1}$.

Definition 10.1. A symmetric motion planner for $X, \mathcal{M}=\left\{F_{i}, s_{i}\right\}$, consists of a collection $F_{1}, \ldots, F_{k}$ (called the local domains of $\mathcal{M}$ ) of subsets of $F(X, 2)$ and a collection of maps $s_{i}: F_{i} \rightarrow P_{1}(X)$ (called the local rules of $\mathcal{M}$ ). This data is required to satisfy:

(a) the local domains form a partition of $F(X, 2)$ (the $F_{i}$ 's cover $F(X, 2)$ and are pairwise disjoint);

(b) each local domain should be a $\mathbb{Z} / 2$-equivariant neighborhood deformation retract of $F(X, 2)$;

(c) each local rule $s_{i}$ should be a continuous $\mathbb{Z} / 2$-equivariant section of $\mathrm{ev}_{1} \mid \mathrm{ev}_{1}^{-1}\left(F_{i}\right) \cdot$

\footnotetext{
${ }^{6}$ Such a property of $X$ follows from $\left[30\right.$ and the inequalities cat $\leq \mathrm{TC} \leq \mathrm{TC}^{S}$. In fact, the considerations after Example 7 in [10] mention the appealing possibility that such a section $s$ exists if and only if $X$ is contractible.
} 
Let us spell out part (b) in Definition 10.1. First, each $F_{i}$ must be stable under the involution in $F(X, 2)$. In addition, $F_{i}$ must have an open neighborhood $U_{i}$ in $F(X, 2)$ which is required to be stable under the involution and to admit a $\mathbb{Z} / 2$ equivariant retraction $r_{i}: U_{i} \rightarrow F_{i}$. We also require that as a map to $F(X, 2), r_{i}$ should be $\mathbb{Z} / 2$-equivariantly deformable to the inclusion $U_{i} \hookrightarrow F(X, 2)$. This last (rather technical, but natural in practical settings) condition captures the essence in 9, Definition 2.1(c)], where the nonsymmetric situation is controlled by requiring the less restrictive hypothesis that each local domain be an ENR. The present requirement is made not only in order to avoid pathological situations, but to have good control on the topology.

Remark 10.2. A symmetric motion planner gives a practical algorithm to approach the programming problem posed at the beginning of the section: just determine the local domain $F_{i}$ containing the given pair of states $(A, B)$, and apply the local rule $s_{i}(A, B)$ in the desired direction. Note that $(B, A)$ lies in $F_{i}$ too, and that $s_{i}(B, A)$ runs the path $s_{i}(A, B)$ in the reverse direction, i.e. $s_{i}(A, B)(t)=s_{i}(B, A)(1-t)$. It is to be observed, however, that such an algorithm will not necessarily satisfy the requirement 3 above: the closure of two (or more) local domains might have a nonempty intersection, a problem inherent to the motion planning task. In fact, Farber proves that in any nonsymmetric motion planner of $X$ (as defined in 9, Definition 2.1]) there are at least $7 \mathrm{TC}(X)+1$ local domains with a nonempty intersection. Furthermore, since by definition there is a nonsymmetric motion planner of $X$ with $\mathrm{TC}(X)+1$ local domains, Farber's bound turns out to be optimal. This leads in 9 to the concept of order of instability of a nonsymmetric motion planner, and to its close connection with the invariant TC. The author hopes to deal elsewhere with a possible symmetric analogue of this phenomenon. As a preliminary step, it is next shown that $\mathrm{TC}^{S}(X)$ is a sharp lower bound for the number of local rules of symmetric motion planners for $X$.

Theorem 1.3 is a direct consequence of Propositions 10.3 and 10.4 below, which, in turn, are proved with arguments inspired by the proof of part (1) of Theorem 6.1 in 9 .

Proposition 10.3. The number of local domains in any symmetric motion planner of $X$ is bounded from below by $\operatorname{TC}^{S}(X)$.

Proof. Let $F \subset F(X, 2)$ be a local domain of a given symmetric motion planner $\mathcal{M}$ with $k$ local rules, and let $s: F \rightarrow P_{1}(X)$ be the local rule corresponding to $F$. Choose an open neighborhood $U$ of $F$ which is stable under the involution in $F(X, 2)$ and admits a retraction $r: U \rightarrow F$ which is $\mathbb{Z} / 2$-equivariantly deformable to the inclusion $U \hookrightarrow F(X, 2)$. Under these conditions the image $U^{\prime}$ of $U$ under the projection $F(X, 2) \rightarrow B(X, 2)$ is open and, as shown below, there is a continuous $\mathbb{Z} / 2$-equivariant section $\sigma: U \rightarrow P_{1}(X)$ for $\mathrm{ev}_{1}$. Granting this and passing to orbit spaces, $\sigma$ determines a continuous section $\sigma^{\prime}$ for $\mathrm{ev}_{2}$ on $U^{\prime}$. When this construction is performed over each of the local domains of $\mathcal{M}$, there results an open cover $U_{1}^{\prime}, \ldots, U_{k}^{\prime}$ of $B(X, 2)$, where each $U_{i}^{\prime}$ admits a local section $\sigma_{i}^{\prime}$ for $\mathrm{ev}_{2}$. Thus $k \geq \operatorname{TC}^{S}(X)$, as asserted.

In order to define the required section $\sigma$ at a pair $(x, y) \in U$, consider the path $H_{x, y}:[0,1] \rightarrow F(X, 2)$ given by $H_{x, y}(t)=H(x, y, t)$, where $H: U \times[0,1] \rightarrow F(X, 2)$

\footnotetext{
${ }^{7}$ In this bound, the " +1 " term comes from our chosen normalization for the genus of a fibration.
} 
is a fixed $\mathbb{Z} / 2$-equivariant homotopy between the inclusion $U \hookrightarrow F(X, 2)$ (at $t=0$ ) and the retraction $r: U \rightarrow F$ (at $t=1$ ). Under these conditions, set $\sigma(x, y)$ to be the concatenation

$$
\sigma(x, y)=\gamma_{x, y} \cdot s\left(\gamma_{x, y}(1), \delta_{x, y}(1)\right) \cdot \bar{\delta}_{x, y},
$$

where $\bar{\delta}_{x, y}$ is the path $\delta_{x, y}$ in reverse, and $\gamma_{x, y}, \delta_{x, y}:[0,1] \rightarrow F(X, 2)$ are the components of $H_{x, y}=\left(\gamma_{x, y}, \delta_{x, y}\right)$. The hypothesis on $H$ means that $\gamma_{x, y}=\delta_{y, x}$ and $\delta_{x, y}=\gamma_{y, x}$, so that $\sigma$ is equivariant, as required.

Proposition 10.4. If $X$ is a smooth manifold, then there is a symmetric motion planner of $X$ with $\mathrm{TC}^{S}(X)$ local rules.

Proof. Let $V_{1}, \ldots, V_{k}$ be an open cover of $B(X, 2)$ with local sections $\sigma_{i}: V_{i} \rightarrow$ $P_{2}(X)$ of $\mathrm{ev}_{2}$, where $k=\mathrm{TC}^{S}(X, 2)$. The assumption $k>1$ can safely be made in view of [10, Lemma 8]. Choose a smooth partition of unity

$$
f_{i}: B(X, 2) \rightarrow[0,1], \quad i=1, \ldots, k,
$$

subordinate to the cover. Using Sard's Theorem it is possible to show 8 the existence of numbers $c_{1}, \ldots, c_{k} \in(0,1)$ satisfying $\sum c_{i}=1$ and such that each $c_{i}$ is a regular value of $f_{i}$. For $i=1, \ldots, k$, consider the subspaces $G_{i}$ of $B(X, 2)$ consisting of sets $\{x, y\} \in B(X, 2)$ satisfying $f_{i}(\{x, y\}) \geq c_{i}$ and $f_{j}(\{x, y\})<c_{j}$ for $j<i$. One checks that each $G_{i}$ is a submanifold (with possibly nonempty boundary) of the corresponding $V_{i}$, and that the collection $\left\{G_{1}, \ldots, G_{k}\right\}$ is a partition of $B(X, 2)$. Pick a tubular (normal) neighborhood $N_{i}$ of $G_{i}$ in $V_{i}$ (so that $N_{i}$ contains $G_{i}$ as a deformation retract). Let $\pi: F(X, 2) \rightarrow B(X, 2)$ be the canonical projection and, for $i=1, \ldots, k$, considering the inverse images $F_{i}=\pi^{-1}\left(G_{i}\right)$, a partition of $F(X, 2)$, and the corresponding open neighborhoods $U_{i}=\pi^{-1}\left(N_{i}\right)$, all of which are stable under the involution in $F(X, 2)$. As explained in [10, Lemma 8], each restricted section $\sigma_{i \mid N_{i}}$ determines a continuous $\mathbb{Z} / 2$-equivariant section $s_{i}: U_{i} \rightarrow P_{1}(X)$ of $\mathrm{ev}_{1}$. Therefore, to conclude the proof, it remains to find $\mathbb{Z} / 2$-equivariant retractions $r_{i}: U_{i} \rightarrow F_{i}$ which are $\mathbb{Z} / 2$-equivariantly deformable to the corresponding inclusions $U_{i} \hookrightarrow F(X, 2)$. This is done in the next paragraph by a standard homotopy lifting argument for the covering projection $\pi: F(X, 2) \rightarrow B(X, 2)$.

For each $i=1, \ldots, k$, choose a homotopy $H_{i}: N_{i} \times[0,1] \rightarrow B(X, 2)$ deforming the inclusion $H_{i}(-, 0): N_{i} \hookrightarrow B(X, 2)$ to a retraction $H_{i}(-, 1): N_{i} \rightarrow G_{i}$. The homotopy lifting property of $\pi$ applied to the commutative diagram

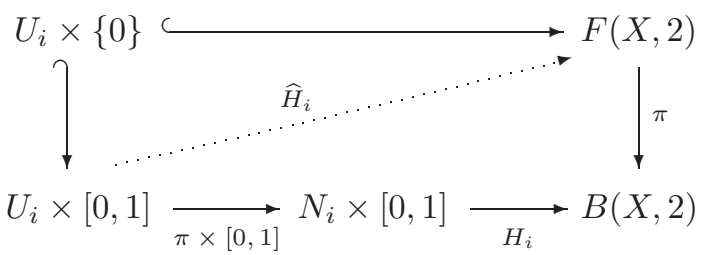

yields the dotted homotopy $\widehat{H}_{i}: U_{i} \times[0,1] \rightarrow F(X, 2)$ compatible with $H_{i}$ under $\pi$, and deforming the inclusion $\widehat{H}_{i}(-, 0): U_{i} \hookrightarrow F(X, 2)$ to the required retraction

\footnotetext{
${ }^{8}$ The author thanks Peter Landweber for pointing out the need for the condition $k>1$, and for explaining the details of this assertion.
} 
$\widehat{H}_{i}(-, 1): U_{i} \hookrightarrow F_{i}$. To see that $\widehat{H}_{i}$ is $\mathbb{Z} / 2$-equivariant, fix a point $(a, b) \in U_{i}$ and observe that, if $\widehat{H}_{i}(a, b, t)=(x, y)$ for some $t \in[0,1]$, then $\widehat{H}_{i}(b, a, t) \in\{(x, y),(y, x)\}$ is forced. We need to see that $\widehat{H}_{i}(b, a, t)=(y, x)$ must in fact be the case. To this end, note that $\widehat{H}_{i}(a, b,-)$ and $\widehat{H}_{i}(b, a,-)$ are two paths $[0,1] \rightarrow F(X, 2)$, starting respectively at $(a, b)$ and $(b, a)$, and projecting under $\pi$ to the same path. So, by the unique path lifting property of $\pi, \widehat{H}_{i}(a, b,-)$ and $\widehat{H}_{i}(b, a,-)$ are point-wise different.

The paper closes by exploiting the main idea in [15] in order to sketch a 5-localrules symmetric motion planner for any autonomous robot whose state space is $\mathrm{P}^{3}=\mathrm{SO}(3)$.

Start by considering the standard Euclidean charts

$$
U_{i}=\left\{\left[x_{0}, \ldots, x_{4}\right] \in \mathrm{P}^{4} \mid \sum_{j} x_{j}^{2}=1 \text { and } x_{i} \neq 0\right\}, \quad 0 \leq i \leq 4,
$$

of $\mathrm{P}^{4}$, and the corresponding local sections $s_{i}: U_{i} \rightarrow S^{4}$ of the canonical projection $q: S^{4} \rightarrow \mathrm{P}^{4}$, where

$$
s_{i}\left(\left[x_{0}, \ldots, x_{4}\right]\right)=\frac{\left|x_{i}\right|}{x_{i}}\left(x_{0}, \ldots, x_{4}\right) .
$$

According to [28], the formula

$$
f\left(\left[z_{0}, z_{1}\right]\right)=\frac{\left(z_{0}^{2}, z_{1}^{2}, \Re\left(z_{0} z_{1}\right)\right)}{\sqrt{1-\left|z_{0} z_{1}\right|^{2}}-\Im\left(z_{0} z_{1}\right)}
$$

determines an explicit topological embedding $f: \mathrm{P}^{3} \subset \mathbb{R}^{5}$, where $\Re(\omega)$ and $\Im(\omega)$ stand, respectively, for the real and imaginary parts of a complex number $\omega$, and where an element in $S^{3}$ has been represented by a pair of complex numbers $z_{i}$ $(i=0,1)$ with $\left|z_{0}\right|^{2}+\left|z_{1}\right|^{2}=1$. Using Haefliger's formula (3), (40) gives an explicit $\mathbb{Z} / 2$-equivariant map $H: F\left(\mathrm{P}^{3}, 2\right) \rightarrow S^{4}$ determining the pull-back diagram

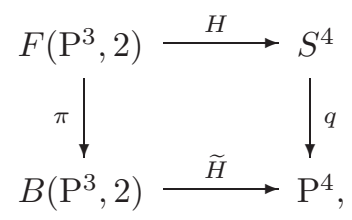

where $\pi$ stands for the canonical projection. In these terms, each section (39) pulls back to an explicit local section $\sigma_{i}: \widetilde{H}^{-1}\left(U_{i}\right) \rightarrow F\left(\mathrm{P}^{3}, 2\right)$ for $\pi$. For instance, $\widetilde{H}^{-1}\left(U_{0}\right)$ consists of those $\left\{\left[z_{0}, z_{1}\right],\left[\omega_{0}, \omega_{1}\right]\right\} \in B\left(\mathrm{P}^{3}, 2\right)$ such that

$$
\left(\sqrt{1-\left|\omega_{0} \omega_{1}\right|^{2}}-\Im\left(\omega_{0} \omega_{1}\right)\right) \Re\left(z_{0}^{2}\right)-\left(\sqrt{1-\left|z_{0} z_{1}\right|^{2}}-\Im\left(z_{0} z_{1}\right)\right) \Re\left(\omega_{0}^{2}\right) \neq 0
$$

whereas

$\sigma_{0}\left(\left\{\left[z_{0}, z_{1}\right],\left[\omega_{0}, \omega_{1}\right]\right\}\right)= \begin{cases}\left(\left[z_{0}, z_{1}\right],\left[\omega_{0}, \omega_{1}\right]\right), & \text { left-hand side of (41) is positive; } \\ \left.\left[\omega_{0}, \omega_{1}\right],\left[z_{0}, z_{1}\right]\right), & \text { left-hand side of (41) is negative. }\end{cases}$ 
But according to [15, Propositions 2.1 and 2.2] there is a well-defined commutative diagram

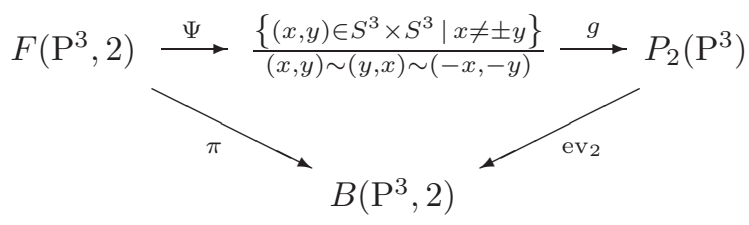

where horizontal maps are explicitly given by

$$
\Psi([x],[y])=\left[\frac{x+y}{\|x+y\|}, \frac{x-y}{\|x-y\|}\right]
$$

and

$$
g([x, y])(t)=\left[\frac{t y+(1-t) x}{\|t y+(1-t) x\|}\right]
$$

for $x, y \in S^{3}$. (Note that a typo occurs in [15] four lines above formula (5), as well as 7 lines below formula (13): the inequality sign in the definition of $\widetilde{\Delta}$ should be replaced by an equality sign.) Therefore, on the open cover

$$
\left\{\widetilde{H}^{-1}\left(U_{0}\right), \ldots, \widetilde{H}^{-1}\left(U_{4}\right)\right\},
$$

the composites $g \circ \Psi \circ \sigma_{i}$ for $i=0, \ldots, 4$ give 5 explicit local sections for $\mathrm{ev}_{2}$, and the required symmetric motion planner is then described by the proof of Proposition 10.4

The above process has only one nonconstructive component, namely, no suitable smooth partition of unity (38) is explicitly given. But a closer look at the proof of Proposition 10.4 shows that the critical goal is to refine (42) to a pair-wise disjoint cover by submanifolds of $B\left(\mathrm{P}^{3}, 2\right)$. The author encourages readers interested in implementing this symmetric motion planner to attempt to provide the missing explicit construction. At any rate, the actual local rules have been explicitly described: after pulling back over $F\left(\mathrm{P}^{3}, 2\right) \rightarrow B\left(\mathrm{P}^{3}, 2\right)$, these are given by restrictions of the composites $g \circ \Psi \circ \sigma_{i}$.

\section{ACKNOWLEDGMENTS}

The author wishes to thank Fred Cohen and Peter Landweber for many useful suggestions for computing the integral cohomology ring of the unordered configuration space of two distinct points in $\mathrm{P}^{m}$, a central object for the purposes of Section 3. Cohen's suggestion of using the Bockstein spectral sequence approach eventually led the author to the calculations described at the end of Examples 8.9. clarifying a subtle point in the main Cartan-Leray-Serre spectral sequence calculation of Section 9. The author wishes to specially thank Peter Landweber for carefully reading this manuscript and for many fine suggestions throughout the

development of this paper. In particular, Section 10 arose from an observation of Landweber on an earlier version of Theorem 1.4

\section{REFERENCES}

[1] L. Astey, "Geometric dimension of bundles over real projective spaces", Quart. J. Math. Oxford Ser. (2) 31 (1980) 139-155. MR576333(81j:55004)

[2] L. Astey, "A cobordism obstruction to embedding manifolds", Illinois J. Math. 31 (1987) 344-350. MR882119 (88a:57056) 
[3] D. R. Bausum, "Embeddings and immersions of manifolds in Euclidean space", Trans. Amer. Math. Soc. 213 (1975) 263-303. MR0474330 (57:13976)

[4] M. C. Crabb, Z/2-homotopy theory, London Mathematical Society Lecture Note Series 44. Cambridge University Press, Cambridge-New York, 1980. MR591680 (83m:55010)

[5] D. M. Davis, "A strong nonimmersion theorem for real projective spaces", Ann. of Math. (2) 120 (1984) 517-528. MR769162 (87b:57021)

[6] D. M. Davis, "Table of immersions and embeddings of real projective spaces", available from http://www. lehigh.edu/ dmd1/immtable

[7] L. Evens, The Cohomology of Groups, Oxford Mathematical Monographs. Oxford Science Publications. The Clarendon Press, Oxford University Press, New York, 1991. MR 1144017 (93i:20059)

[8] M. Farber, "Topological complexity of motion planning", Discrete Comput. Geom. 29 (2003) 211-221. MR,1957228(2004c:68132)

[9] M. Farber, "Instabilities of robot motion", Topology Appl. 140 (2004) 245-266. MR2074919 (2005g:68166)

[10] M. Farber and M. Grant, "Symmetric motion planning", in Topology and Robotics, Contemp. Math. 438, Amer. Math. Soc., Providence, RI (2007) 85-104. MR2359031 (2008i:55016)

[11] M. Farber, S. Tabachnikov, and S. Yuzvinsky, "Topological robotics: motion planning in projective spaces", Int. Math. Res. Not. 34 (2003) 1853-1870. MR1988783(2004i:55005)

[12] S. Feder, "The reduced symmetric product of projective spaces and the generalized Whitney theorem", Illinois J. Math. 16 (1972) 323-329. MR0296966 (45:6025)

[13] Y. Félix and D. Tanré, "The cohomology algebra of unordered configuration spaces", J. London Math. Soc. (2) 72 (2005) 525-544. MR2156668 (2006d:55017)

[14] S. Gitler and D. Handel, "The projective Stiefel manifolds I", Topology 7 (1968) 39-46. MR.0220307 (36:3373a)

[15] J. González and P. Landweber, "Symmetric topological complexity of projective and lens spaces", Algebr. Geom. Topol. 9 (2009) 473-494. MR2491582 (2009k:57045)

[16] J. González and P. S. Landweber, "The integral cohomology of configuration spaces of pairs of points in real projective spaces", in preparation.

[17] T. G. Goodwillie, J. R. Klein, and M. S. Weiss, "Spaces of smooth embeddings, disjunction and surgery", in Surveys on Surgery Theory, Ann. of Math. Stud. 149 (2001) 221-284. MR $1818775(2001 \mathrm{~m}: 57053)$

[18] T. G. Goodwillie, J. R. Klein, and M. S. Weiss, "A Haefliger style description of the embedding calculus tower", Topology 42 (2003) 509-524. MR.1953238 (2003m:57062)

[19] T. G. Goodwillie and M. S. Weiss, "Embeddings from the point of view of immersion theory, Part II", Geom. Topol. 3 (1999) 103-118. MR.1694808(2000c:57055b)

[20] A. Haefliger, "Points multiples d'une application et produit cyclique réduit", Amer. J. Math. 83 (1961) 57-70. MR0120655(22:11404)

[21] D. Handel, "An embedding theorem for real projective spaces", Topology 7 (1968) 125-130. MR 0227998 (37:3582)

[22] A. Hatcher, Algebraic Topology, Cambridge University Press, Cambridge, 2002. MR1867354 (2002k:55001)

[23] J. W. Milnor and J. D. Stasheff, Characteristic Classes, Annals of Mathematics Studies No. 76, Princeton University Press, Princeton NJ, 1974. MR0440554 (55:13428)

[24] B. A. Munson, "Embeddings in the 3/4 range", Topology 44 (2005) 1133-1157. MR 2168572 (2006d:57043)

[25] E. Rees, "Embeddings of real projective spaces", Topology 10 (1971) 309-312. MR 0288778 (44:5974)

[26] A. S. Schwarz, "The genus of a fiber space", Amer. Math. Soc. Transl. Ser. 255 (1966) 49-140.

[27] S. Stolz, "The level of real projective spaces", Comment. Math. Helv. 64 (1989) 661-674. MR:1023002 (91e:55017)

[28] G. Vranceanu and T. Ganea, "Topological embeddings of lens spaces", Proc. Cambridge Philos. Soc. 57 (1961) 688-690. MR0124908 (23:A2217)

[29] M. S. Weiss, "Embeddings from the point of view of immersion theory, Part I", Geom. Topol. 3 (1999) 67-101. MR1694812(2000c:57055a)

[30] G. W. Whitehead, "On mappings into group-like spaces", Comment. Math. Helv. 28 (1954) 320-328. MR0065927 (16:505c) 
[31] T. Yasui, "Note on the enumeration of embeddings of real projective spaces", Hiroshima Math. J. 3 (1973) 409-418. MR0339220(49:3982)

[32] T. Yasui, "Note on the enumeration of embeddings of real projective spaces II", Hiroshima Math. J. 6 (1976) 221-225. MR0436171 (55:9121)

[33] G. Yo (Yueh Ching-chung), "Cohomology mod $p$ of deleted cyclic product of a manifold", Sci. Sinica 12 (1963) 1779-1794. MR0182003(31:6228)

Departamento de Matemáticas, Centro de Investigación y de Estudios Avanzados del Instituto Politecnico Nacional, Apartado Postal 14-740 México City, C.P. 07000, MÉXICO

E-mail address: jesus@math.cinvestav.mx 